\title{
How Covariant Closed String Theory Solves a Minimal Area Problem
}

\author{
Barton Zwiebach * \\ Center for Theoretical Physics, Laboratory for Nuclear Science, and Department of Physics, \\ Massachusetts Institute of Technology, Cambridge, MA 02139, USA
}

Received February 7, 1990

\begin{abstract}
For a given genus $g$ Riemann surface with $n \geqq 0$ punctures ( $n \geqq 3$ for $g=0$ ) we consider the problem of finding the metric of minimal area under the condition that the length of any nontrivial closed curve be greater or equal to $2 \pi$. The minimal area metrics are found for the case of all punctured genus zero surfaces and for many of the higher genus surfaces both with and without punctures. These metrics are induced by Jenkins-Strebel quadratic differentials. They arise from the string diagrams corresponding to restricted Feynman graphs of a closed string field theory action containing classical and quantum restricted polyhedra.
\end{abstract}

\section{Introduction and Summary}

During the last year considerable progress was made on the formulation of covariant closed string field theory. The classical field theory turned out fully nonpolynomial and its interactions were determined completely [SaZw, KKS]. It was proven that no purely cubic theory with a symmetric vertex could ever be satisfactory [SoZw 1], and, modulo a technical assumption, it was established that covariant closed string field theory exists to all orders in the loop expansion [SoZw 2]. Finally, the various terms entering into the classical action and the gauge transformation of the string field have been written quite explicitly in [KS].

The determination of the string interactions and string diagrams for the classical closed string field theory was no simple matter. The most useful tool was found to be a theorem of Strebel [St 1, St 2] which was used to understand how to construct Riemann surfaces as contact interactions [SaZw]. This theorem, however, could not be used to understand the surfaces that arise via Feynman diagrams with internal lines. It was nevertheless possible to determine the complete set of interactions [SaZw, KKS] and to give an argument that suggested

* Supported in part by funds provided by the U.S. Department of Energy (D.O.E.) under contract \# DE-AC02-76ER03069 
strongly that the classical theory generated correctly all punctured Riemann spheres [KKS]. The argument in [KKS] actually established that the (apparent) boundaries of the moduli space regions generated by the various Feynman graphs can be paired. Provided the two regions that share each boundary lie on opposite sides of the boundary (an intuitive argument for this was given in [KKS]) the matching of boundaries guarantees BRST invariance. This argument, however does not establish that each surface is produced once and only once. It is possible to imagine that a given Feynman graph might produce some surfaces more than once, or that there could be some overlap between regions that share a boundary, since it is not clear that all points of a region have to be in one side of the boundary. ${ }^{1}$ It would be desirable to rule out these possibilities and to establish conclusively the correct covering of moduli space. The physical motivation for the present work was to prove that indeed the tree diagrams of the closed string field theory do cover once and only once the moduli spaces of the $n$-punctured Riemann spheres. This proof will be given, and is expected to provide a firm basis for future development of the theory. Another important physical issue is that of the higher genus string diagrams. In fact, the understanding of the tree level string diagrams do give a strong suggestion for a canonical definition of the higher genus diagrams both for surfaces with and without punctures. (A brief summary of the results of the present paper, with emphasis on physics issues, can be found in $[\mathrm{Zw}]$.)

The future development of closed string field theory will depend to a large degree on finding a useful mathematical language. Such language is necessary in order to write down the action in an elegant way. The present results may be a step in this direction. The principle of minimal area, although admittedly still tied to the perturbative definition of the theory, is very suggestive. At a more concrete level we will see that it will be necessary to construct new existence and uniqueness theorems for quadratic differentials, extending previous results by Jenkins and Strebel. The idea of reduced area, which is a useful extension of the idea of area suitable for punctured surfaces will be introduced.

The usual extremal metric problem consists of picking an admissible set of curves on a surface (nonintersecting, nonhomotopic curves), requiring that lengths of curves homotopic to these be greater or equal to some fixed constants, and finding the metric of minimal area. This metric always arises from a quadratic differential with closed trajectories, namely, a Jenkins-Strebel (JS) quadratic differential. It will be seen that the string diagrams are the solutions of the following generalized minimal area problem:

Minimal Area Problem. Given a genus $g$ Riemann surface $R$ with $n \geqq 0$ punctures ( $n \geqq 3$ for $g=0$ ) find the metric of minimal (reduced) area under the condition that the length of any nontrivial homotopy closed curve be greater or equal to $2 \pi$.

This problem is very different from the usual minimal area problem because this time one does not fix a particular admissible set of curves, one requires that all nontrivial curves (including those homotopic to punctures) must be larger than some constant, and the set of all curves is not an admissible set of curves! It is very important to realize that just from the statement of the problem the minimal area metric is expected to be modular invariant, factorizable, and independent of the labeling of the punctures. It should be modular invariant because the condition that all curves be longer than a fixed constant is invariant under modular

${ }^{1}$ I thank S. Carlip who raised this type of possibilities to me 
transformations. It should be factorizable, roughly speaking, because of the additivity of the area, if a minimal area surface is made out of two surfaces joined by a long tube, the two surfaces must be of minimal area. The problem is also manifestly independent of the labeling of the punctures. These three properties are hallmarks of a covariant field theory formulation, as explained in [SoZw 2]. In order to have off-shell amplitudes we do not only need a metric, but also a canonical definition of coordinates around punctures. These coordinates are easily found if the metric arises from a quadratic differential with second order poles with negative residues at the punctures [SoZw 2].

The results of this paper can be summarized making reference to the following string action

$$
S=\int \mathscr{L}, \mathscr{L}=\Phi Q \Phi+\sum_{n \geqq 3} \mathscr{V}_{0, n} \Phi^{n}+\sum_{g \geqq 1 ; n} \hbar^{g} \mathscr{V}_{g, n} \Phi^{n}
$$

Here, schematically, the term $\Phi Q \Phi$ represents the kinetic term, and the vertices $\mathscr{V}_{0, n}$ are the restricted polyhedra of the classical field theory [SaZw, KKS] (precise definitions are given in Sect. 3). It is established that the tree level Feynman graphs or string diagrams of the classical closed string field theory give the solution of the minimal area problem posed above for Riemann spheres with three or more punctures. This result is used to show the correct covering of moduli space (without overcounting or undercounting) for the classical theory.

It is also shown, however, that the naive Feynman graphs built with the classical interactions eventually miss higher genus surfaces. It thus seems very likely that the complete quantum action will require new interactions. Reasonable candidates are given by the restricted quantum polyhedra denoted by $\mathscr{V}_{g, n}$. Their contribution to the action is accompanied by powers of $\hbar$ as shown above. Using naive Feynman rules the above action will not miss any surface, but there will be overcounting. There is a very interesting subset of Feynman graphs that do not give overcounting (but apparently undercount). These are restricted Feynman graphs, defined to be graphs following from the above action, but satisfying the additional constraint that no closed curve be shorter than $2 \pi$. This constraint only affects higher genus diagrams. The restricted Feynman graphs generate subspaces of every moduli space $\mathscr{M}_{g, n}$ of the higher genus surfaces with or without punctures. It is shown that the restricted Feynman graphs do give the metric of minimal area, thus providing the solution for the above minimal area problem for a large fraction of the surfaces of higher genus. Since the Feynman graphs of the above field theory define JS quadratic differentials, it follows that the extremal metric arises from a JS quadratic differential. The restricted Feynman graphs construct a system of factorizable quadratic differentials, namely a system which is consistent with degeneration of surfaces. From the viewpoint of string field theory the above results suggest that the final formulation will be able to deal correctly not only with punctured surfaces, but also with the unpunctured ones (vacuum graphs).

The main open problem left in this paper is finding the string diagrams for the surfaces that are not generated by the restricted Feynman graphs. It seems natural that they should also be given by the metric solving the minimal area problem. But we do not know what the minimal area metric is for the missing surfaces. Once these are found it will be possible to study if the above quantum action is really the complete action. With the results of the present paper, the classical part has now been proven to generate correctly the required moduli spaces. The quantum part, suggested by some of our results, is still quite tentative. Our results imply an 
(apparently new) cell decomposition of the moduli spaces of punctured Riemann spheres. Each cell is generated by a different tree level Feynman graph. A complete understanding of the minimal area problem would possibly extend this result to all moduli spaces of punctured and unpunctured Riemann surfaces.

Having summarized the main results, let us turn to the detailed contents of this work. The logical steps in developing our results were the following. There were theorems by Strebel [St 2] showing how to construct punctured surfaces with quadratic differentials whose characteristic ring domains are just punctured disks, and no internal annuli. There were also theorems by Jenkins [Je] on how to construct surfaces without punctures with internal annuli. Here the number of annuli used can be (essentially) fixed by specifying some curves on the surface homotopic to the desired annuli. The Strebel construction is modular invariant, since it requires no specification of curves on the surface, but the Jenkins construction is not. Since the diagrams of closed string field theory have punctured disks and intermediate annuli it was necessary to develop a new existence and uniqueness theorem for quadratic differentials combining both of the above constructions. This is done in Sect. 5; the resulting quadratic differentials, having both punctured disks and internal annuli, also need the specification of curves homotopic to the desired annuli. In order to obtain quadratic differentials that do not depend on a choice of curves (or annuli) a generalized extremal problem is proposed (Sect. 7). One searches over all possible choices of a set of curves (an infinite number), each set furnishing a quadratic differential, and picks the quadratic differential with largest norm (under the condition that all ring domains have closed trajectories of length $2 \pi$ ). It will be shown that for Riemann spheres this extremal problem furnishes a unique quadratic differential, precisely that defining the string diagram.

This paper is organized as follows. In Sect. 2 various notions necessary for our proofs are developed. The idea of reduced area, which is a regularized version of the area of a punctured surface is introduced. It is shown that a metric of minimal reduced area is unique. This is an essential property which will allow us to show the uniqueness of the string diagram in Sect. 7. The basic extremal properties of Jenkins-Strebel quadratic differentials are reviewed for the case of annuli, and discussed for the slightly more delicate case of punctured disks. Finally the relation between admissible sets of curves, the structure of Feynman graphs and quadratic differentials is explained.

In Sect. 3 the quadratic differentials relevant to the field theory of closed strings are studied. We first review and streamline the basic ideas in the construction of the classical field theory and the definitions of polyhedra and restricted polyhedra. Polyhedra associated to punctured Riemann surfaces are called classical polyhedra, and polyhedra associated with higher genus punctured surfaces are called quantum polyhedra. An existence and uniqueness theorem for quadratic differentials is proposed (Theorem 3.2). While its proof is postponed to Sect. 7; it is shown that it would guarantee the correct covering of the moduli spaces of Riemann spheres by the classical theory.

In Sect. 4 we review and slightly reformulate for our purposes the relevant existence and uniqueness theorems of Jenkins and Strebel mentioned above. This review is necessary to set up the proof in Sect. 5 of the new existence and uniqueness theorem for quadratic differentials with closed trajectories and both punctured disks and internal annuli. In Sect. 6 the newly established theorem is 
used to explain some simple facts about the lack of overcounting by some special Feynman graphs. A detailed discussion of the four-punctured sphere is very illustrative and provides motivation for the generalized extremal problem. It is also shown why the naive tadpole graph overcounts.

In Sect. 7 a generalized extremal problem that chooses among many candidate quadratic differentials the one with maximal norm is proposed. It is shown that the solution of this problem furnishes the unique quadratic differential that satisfies the conditions of Theorem 3.2 establishing in this way the correctness of the classical theory. As a by product we learn that the string diagram is actually the surface of minimal area under the condition that the length of all nontrivial closed curves be greater or equal to $2 \pi$.

In Sect. 8 the possibility that the above minimal area problem also defines the higher genus string diagrams is studied. Restricted quantum polyhedra are shown to be necessary to produce the minimal area metrics for some of the surfaces in $\mathscr{M}_{g, n}$ for sufficiently high $n$ (at fixed $g$ ). The properties of restricted Feynman graphs are studied. Even though they seem to miss surfaces for higher dimensional moduli spaces, it is very curious that the restricted Feynman graphs generate automatically the modular region for the one-loop vacuum graph.

In Sect. 9 we conclude with some comments on open problems.

\section{Basic Notions and Developments}

\subsection{Admissible Metrics and Reduced Area}

In this section the well-known definition of an admissible metric on a Riemann surface will be reviewed. Given an admissible metric one can compute lengths of curves and areas of regions. For the case of punctured surfaces, however, the computation of area is not straightforward. The main objective of this section is to introduce a regularized version of an area, the reduced area appropriate for punctured surfaces. Its key property is that in the same way as one can show that metrics of minimal area are unique, it will be shown that for punctured surfaces metrics of minimal reduced area are also unique. In the next subsection it will be seen that the reduced area, for the case of metrics defined by quadratic differentials, is nothing else than the reduced norm of the quadratic differential.

A metric on a Riemann surface is denoted by the quantity $\varrho|d z|$ defining the length element in some local coordinate $z(=x+i y)$. Since $\varrho$ is real it cannot be a nontrivial analytic object. Special $\varrho$ 's may be the absolute value of analytic objects: $\varrho \sim|h(z)|$. This is the case for metrics arising from abelian or quadratic differentials, but it is not the general case. Under analytic maps $z \rightarrow \tilde{z}$, the length element must be invariant

$$
\varrho|d \tilde{z}|=\varrho|d z| .
$$

It follows from the above equation that the area element $d A$ defined by

$$
d A \equiv \varrho^{2} d x \wedge d y=\varrho^{2} \frac{i}{2} d z \wedge \overline{d z}
$$


is also invariant under conformal mappings since

$$
\begin{aligned}
\tilde{\varrho}^{2} \frac{i}{2} d \tilde{z} \wedge \overline{d \tilde{z}} & =\tilde{\varrho}^{2} \frac{d \tilde{z}}{d z} \frac{\overline{d \tilde{z}}}{d z} \frac{i}{2} d z \wedge \overline{d z} \\
& =\tilde{\varrho}^{2} \frac{|d \tilde{z}|^{2}}{|d z|^{2}} \frac{i}{2} d z \wedge \overline{d z} \\
& =\varrho^{2} \frac{i}{2} d z \wedge \overline{d z},
\end{aligned}
$$

where use was made of Eq. (2.1.1) in the last step.

The definition of metric used here corresponds, in the physics literature, to a choice of Weyl factor. This is clear once the metric is written if the form $d s^{2}$ $=g_{\alpha \beta} d x^{\alpha} d x^{\beta}=\varrho^{2}\left(d x^{2}+d y^{2}\right)$. The function $\varrho$ is the Weyl factor of a flat metric.

Consider an admissible set of curves, namely, a set of nontrivial homotopy and nonintersecting closed curves $\gamma_{k}$ on a Riemann surface $R$. To each curve in this set associate a number $A_{k}>0$. A metric $\varrho(z)|d z|$ is called admissible for the set $A_{k}$, if

$$
\int_{\gamma \sim \gamma_{k}} \varrho|d z| \geqq A_{k},
$$

for any curve $\gamma$ freely homotopic to $\gamma_{k}$, and for all $k$. Namely, in the admissible metric, the length of any curve homotopic to a given curve $\gamma_{k}$ of the admissible set of curves cannot be lower than $A_{k}$.

Suppose the surface $R$ has some punctures $P_{j}$ with $j=1,2, \ldots, n$. Consider curves $\gamma_{j}$ surrounding each of the punctures, and associate to each of these curves the number $A_{j}{ }^{2}$ A metric $\varrho|d z|$ is called admissible for the set $A_{j}$ if

$$
\int_{\gamma \sim \gamma_{j}} \varrho|d z| \geqq A_{j},
$$

for any curve $\gamma$ freely homotopic to $\gamma_{j}$, for all $j$. The physical picture of a metric admissible for the set $A_{j}$ is that of a surface having a semiinfinite tube of circumference greater or equal to $A_{j}$ at each puncture. Indeed, if we use a coordinate $z_{j}$ vanishing at the puncture $P_{j}$, the metric $\varrho$ cannot be finite as $z_{j} \rightarrow 0$, preserving the above length condition. One typically has near $z_{j}=0$ :

$$
\varrho^{2}=\left(\frac{A_{j}}{2 \pi}\right)^{2} \frac{1}{\left|z_{j}\right|^{2}}+\mathcal{O}\left(\left|z_{j}\right|^{-1}\right), \quad\left(z_{j} \rightarrow 0\right),
$$

making it possible to satisfy the length condition near $z_{j}=0$,

$$
\lim _{r \rightarrow 0} \int_{\left|z_{j}\right|=r} \varrho\left|d z_{j}\right|=\frac{A_{j}}{2 \pi} \int \frac{|d z|}{|z|}=A_{j} .
$$

If $\varrho^{2}$ has a singularity stronger than $|z|^{-2}$ near a puncture, the lengths of closed curves surrounding the puncture will diverge as one approaches the puncture. Such metrics, although admissible by definition, are not interesting because they cannot correspond to a metric of minimal area, as it will be seen shortly. The area associated with the metric in (2.1.5) is clearly seen to diverge, since for a given $r$

\footnotetext{
2 We use the notation of [St 1] in which curves homotopic to punctures are labeled by the index $j$ and curves that are not homotopic to punctures are labeled by $k$. The label $i$ is used for both types of curves
} 
small enough one has

$$
\iint_{|z| \geqq r} \varrho^{2} d x d y \sim \frac{A_{j}^{2}}{(2 \pi)^{2}} \iint_{r^{\prime}>r} \frac{d r^{\prime}}{r^{\prime}} d \theta \sim-\frac{A_{j}^{2}}{2 \pi} \ln r .
$$

It is natural to define a regularized version of the area by subtracting this logarithmic divergence for each puncture.

Definition. The reduced area $\mathscr{A}(\varrho)$ for a metric $\varrho|d z|$ admissible for the set $A_{j}$ corresponding to closed curves $\gamma_{j}$ surrounding the punctures $P_{j}$ is given by

$$
\mathscr{A}(\varrho) \equiv \lim _{r \rightarrow 0}\left(\int_{R(r)} \varrho^{2} d x d y++\frac{1}{2 \pi} \ln r \sum A_{j}^{2}\right),
$$

where $R(r)$ denotes the surface obtained excising the disks $\left|z_{j}\right| \leqq r$ from the original surface $R$, and the $z_{j}$ 's are a fixed set of local coordinates at the punctures.

For metrics $\varrho^{2}$ of the type of Eq. (2.1.5) the reduced area will be finite. For metrics with stronger singularities the reduced area will still be infinite. The reduced area of a region of a surface will be understood to be given by Eq. (2.1.8) with the integral extending over the region and with the sum including only the terms corresponding to the punctures that lie inside the region. In this way it follows that the reduced area of a punctured surface is the sum of the reduced areas of any set of disjoint regions that cover the surface (with no punctures lying on the boundaries between the regions). The metric $\varrho$ in (2.1.8) may, of course, satisfy additional length constraints associated with an admissible set of curves.

As is typical in regularized quantities, finite parts can be convention dependent. The reduced area $\mathscr{A}(\varrho)$ has some dependence on the local coordinates at the punctures. Let $\tilde{z}_{j}$ be another set of local coordinates vanishing at the punctures and denote the corresponding reduced area by $\tilde{\mathscr{A}}(\varrho)$. The two sets of local coordinates must be related by $\tilde{z}_{j}=a_{j} z_{j}$, near the punctures, with $a_{j}$ 's constants. Thus, near the punctures, disks $\left|\tilde{z}_{j}\right|=r$, correspond to disks $\left|z_{j}\right|=r /\left|a_{j}\right|$. It follows then from Eq. (2.1.8), by a simple calculation that

$$
\tilde{\mathscr{A}}(\varrho)=\mathscr{A}(\varrho)+\frac{1}{2 \pi} \sum_{j} A_{j}^{2} \ln \left|a_{j}\right| .
$$

Thus under a change of coordinates, the reduced area changes by a metric independent constant. It follows that the statement that a metric has extremal reduced area is coordinate independent.

Let us now show the uniqueness of a metric with minimal reduced area. The following proof is an extension of the proof presented in [St 1, p. 131] establishing that for metrics of finite area, the extremal metric is unique. Consider a Riemann surface $R$ with $n \geqq 0$ punctures, with an admissible set of curves $\gamma_{k}$, and $n$ curves $\gamma_{j}$ homotopic to the punctures. The associated set of constants $A_{i}$ includes the constants $A_{k}$ associated with the curves $\gamma_{k}$, and $n$ constants $A_{j}$ associated to the curves $\gamma_{j}$. We want to show that among all metric admissible for the $A_{i}$ 's, the one with minimal reduced area is unique. Assume there are two metrics $\varrho_{1}$ and $\varrho_{2}$ that minimize the reduced area, namely $\mathscr{A}\left(\varrho_{1}\right)=\mathscr{A}\left(\varrho_{2}\right)=m$. Using the definition of reduced area in Eq. (2.1.8) both for $\varrho_{1}$ and $\varrho_{2}$, one finds, by subtraction that

$$
\lim _{r \rightarrow 0}\left(\left\|\varrho_{1}^{2}\right\|_{R(r)}-\left\|\varrho_{2}^{2}\right\|_{R(r)}\right)=0
$$


where, for brevity, we have defined

$$
\left\|\varrho_{n}^{2}\right\|_{R(r)} \equiv \int_{R(r)} \varrho_{n}^{2} d x d y .
$$

Since both metrics $\varrho_{1}$ and $\varrho_{2}$ are admissible for the $A_{i}$ 's, we must also have:

$$
\int_{\gamma \sim \gamma_{i}} \varrho_{n}|d z| \geqq A_{i},
$$

for all $i$ 's and for $n=1,2$. Consider now the metric $\varrho=\lambda_{1} \varrho_{1}+\lambda_{2} \varrho_{2}$, where $\lambda_{1}$ and $\lambda_{2}$ are real constants satisfying $\lambda_{1}+\lambda_{2}=1$. It follows that

$$
\int_{\gamma \sim \gamma_{i}} \varrho|d z|=\lambda_{1} \int_{\gamma \sim \gamma_{i}} \varrho_{1}|d z|+\lambda_{2} \int_{\gamma \sim \gamma_{i}} \varrho_{2}|d z| \geqq\left(\lambda_{1}+\lambda_{2}\right) A_{i}=A_{i},
$$

and therefore the new metric $\varrho$ is also admissible for the $A_{i}$ 's. Its reduced area, as a consequence must be greater or equal to the minimal possible value $m$;

$$
m \leqq \mathscr{A}(\varrho)=\lim _{r \rightarrow 0}\left(\int_{R(r)}\left(\lambda_{1} \varrho_{1}+\lambda_{2} \varrho_{2}\right)^{2} d x d y+\frac{1}{2 \pi} \ln r \sum_{j=1}^{n} A_{j}^{2}\right) .
$$

Expanding out and using $\mathscr{A}\left(\varrho_{1}\right)=\mathscr{A}\left(\varrho_{2}\right)=m$, one finds

$$
m \leqq \mathscr{A}(\varrho)=\left(\lambda_{1}^{2}+\lambda_{2}^{2}\right) m+2 \lambda_{1} \lambda_{2} \lim _{r \rightarrow 0}\left(\iint_{R(r)} \varrho_{1} \varrho_{2} d x d y+\frac{1}{2 \pi} \ln r \sum_{j=1}^{n} A_{j}^{2}\right) .
$$

Making use of the Schwarz inequality

$$
\iint_{R(r)} \varrho_{1} \varrho_{2} d x d y \leqq\left[\left\|\varrho_{1}^{2}\right\|_{R(r)}\left\|\varrho_{2}^{2}\right\|_{R(r)}\right]^{1 / 2},
$$

and of Eq. (2.1.10), which implies that

$$
\lim _{r \rightarrow 0}\left(\left[\left\|\varrho_{1}^{2}\right\|_{R(r)}\left\|\varrho_{2}^{2}\right\|_{R(r)}\right]^{1 / 2}-\left\|\varrho_{1}^{2}\right\|_{R(r)}\right)=0,
$$

one shows that the term in parentheses in Eq. (2.1.15) satisfies

$$
\lim _{r \rightarrow 0}\left(\int_{R(r)} \varrho_{1} \varrho_{2} d x d y+\frac{1}{2 \pi} \ln r \sum_{j=1}^{n} A_{j}^{2}\right) \leqq m .
$$

If therefore follows from Eq. (2.1.15) that $m \leqq \mathscr{A}(\varrho) \leqq m$, and as a consequence $\mathscr{A}(\varrho)=m$. Back in Eq. (2.1.15) this requires that

$$
\lim _{r \rightarrow 0}\left(\int_{\boldsymbol{R}(r)} \varrho_{1} \varrho_{2} d x d y+\frac{1}{2 \pi} \ln r \sum_{j=1}^{n} A_{j}^{2}\right)=m .
$$

For the above to hold it is necessary that in the limit as $r$ goes to zero the Schwarz inequality becomes an equality:

$$
\lim _{r \rightarrow 0}\left(\left[\left\|\varrho_{1}^{2}\right\|_{R(r)}\left\|\varrho_{2}^{2}\right\|_{R(r)}\right]^{1 / 2}-\iint_{R(r)} \varrho_{1} \varrho_{2} d x d y\right)=0 .
$$

The fact that the Schwarz inequality is saturated implies that $\varrho_{1}=\varrho_{2}$ a.e. (almost everywhere). Indeed using this last equation and Eq. (2.1.17) one can show that $\lim _{r \rightarrow 0} \int_{R(r)}\left(\varrho_{1}-\varrho_{2}\right)^{2}=0$, and this finally shows that $\varrho_{1}=\varrho_{2}$ (a.e.), establishing the uniqueness of the metric of minimal reduced area. 


\subsection{Quadratic Differentials, Annuli and Punctured Disks}

In this section after reviewing briefly some notions of quadratic differentials ${ }^{3}$ the basic extremal properties of quadratic differentials are discussed. The case of a regular annulus, namely one with finite modulus, is treated first. Here the metric associated with a quadratic differential gives the lowest possible area among all metrics satisfying a suitable length condition. Then we consider in detail the slightly more subtle case of punctured disks. These correspond to annuli in which the inner radius goes to zero, making the modulus infinite. Here the appropriate notion is that of reduced modulus [Te]. Most of our effort will go into showing that the notion of reduced area introduced in the previous section fits naturally with that of reduced modulus. It will be seen that, in analogy with the case for annuli, the metric associated with a quadratic differential is, among all metrics satisfying a suitable length condition, the one with minimal reduced area. All of the above ideas will be useful in the later sections of this paper.

A quadratic differential $\varphi$ on a Riemann surface $R$ is a set of function elements $\phi_{i}\left(z_{i}\right)$, meromorphic in the local coordinates $z_{i}\left(=x_{i}+i y_{i}\right)$ with the transformation property

$$
\phi_{i}\left(z_{i}\right)\left(d z_{i}\right)^{2}=\phi_{j}\left(z_{j}\right)\left(d z_{j}\right)^{2}
$$

under a change of local coordinates. The function element $\phi_{i}$ is called the representation of the quadratic differential in terms of the local coordinate $z_{i}$. To a quadratic differential one can associate the length element $\left|\phi_{i}\left(z_{i}\right)\right|^{1 / 2}\left|d z_{i}\right|$. It follows from Eq. (2.2.1) that the length element is invariant under conformal maps, in other words, the metric $\varrho=\left|\phi_{i}\right|^{1 / 2}$ satisfies Eq. (2.1.1). In a similar way one associates to a quadratic differential an area element $d A=\left|\phi_{i}\left(z_{i}\right)\right| d x_{i} \wedge d y_{i}$. This area element is also conformally invariant since the argument given in Eq. (2.1.3) applies. Thus a quadratic differential defines a metric (the $\phi$-metric) which can be used to compute lengths and areas.

A horizontal trajectory of a quadratic differential is a curve along which $\phi$ $(d z)^{2}$ is real and positive. Quadratic differentials with closed horizontal trajectories, called Jenkins-Strebel (JS) quadratic differentials, are those for which the nonclosed trajectories cover a set of measure zero on the surface. A JS quadratic differential decomposes a surface into characteristic ring domains, the maximal ring domains swept by the closed trajectories. These ring domains can be annuli or punctured disks.

Consider now an annulus, as shown in Fig. 1. In the $z$-plane it appears as the rectangular region bounded by vertical lines through $z=0$ and $z=a$ (identified) and horizontal lines through $z=0$ and $z=i b$. The modulus $M$ of the annulus is given by $M=b / a$ and is a conformal invariant. The JS-quadratic differential $\varphi=\phi(z)(d z)^{2}=(d z)^{2}$ on the annulus gives rise to a metric for which the rectangular region has sides of lengths $a$ and $b$, area $a b=a^{2} M$, and for which any closed curve, such as $\hat{\gamma}$ has length greater or equal to $a$. Define the metric $\varrho|d z|$ to be admissible if for any closed curve $\gamma$ homotopic to $\hat{\gamma}$ one has that

$$
\int_{\gamma \sim \hat{\gamma}} \varrho|d z| \geqq a .
$$

${ }^{3}$ For a complete treatment the reader may consult $[\mathrm{St} 1, \mathrm{Ga}]$, in the mathematical literature. Explanations that have appeared in the physics literature [GiMa, $\mathrm{Ca}, \mathrm{SaZw}$ ], may also be useful 


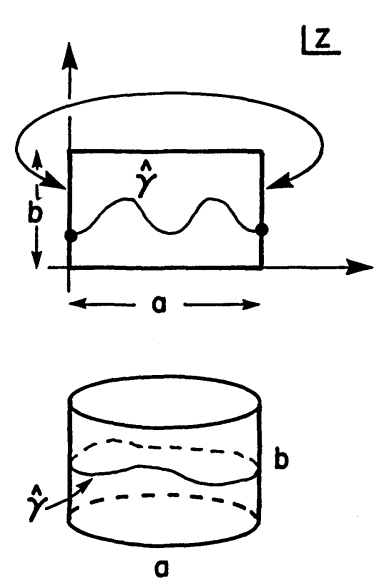

(a)

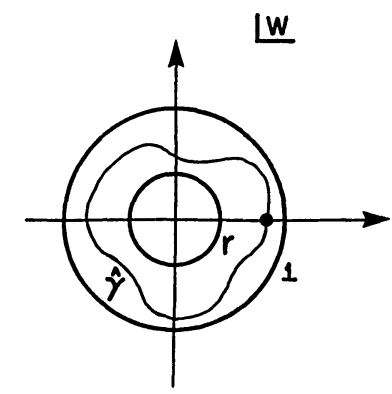

(b)

Fig. 1. a An annulus presented as a rectangular region in the $z$-plane. The height of the annulus is $b$ and its circumference is $a$. Its modulus is given by $M=b / a$. b The annulus presented (in the standard form) as the region $r \leqq|w| \leqq 1$. The curve $\hat{\gamma}$ is a noncontractible simple closed curve

Note that the $\phi$-metric $|\phi|^{1 / 2}|d z|=|d z|$ is admissible. One can show (see, for example [St 1]) that among all admissible metrics $\varrho$, the $\phi$-metric is that of lowest area

$$
\|\phi\|_{R} \equiv \iint_{R}|\phi| d x d y=a^{2} M \leqq \iint_{R} \varrho^{2} d x d y .
$$

The annulus can be mapped into the $w$-plane as shown in Fig. 1 by the relation $w=\exp (2 \pi i z / a)$. The annulus now extends from $|w|=1$ to $|w|=r$, where $r$ is defined by $\ln (1 / r)=2 \pi b / a$. It follows that the modulus of the annulus is given by $M=\frac{1}{2 \pi} \ln (1 / r)$. In the $w$-coordinates the JS quadratic differential reads

$$
1 \cdot(d z)^{2}=\phi(w)(d w)^{2} \rightarrow \phi(w)=-\frac{a^{2}}{(2 \pi)^{2}} \frac{1}{w^{2}} .
$$

An inequality analogous to that in (2.2.3) holds in this presentation, due to the conformal invariance of each term.

Let us now turn to the case of punctured disks. Consider the punctured disk $D_{j}$ in the $z_{j}$-plane bounded by the curve $\Gamma$ and with the puncture at $z_{j}=0$, as illustrated in Fig. 2. This punctured disk can be mapped into a circular disk of some fixed radius $r_{j}$ in the $\xi_{j}$-plane if we require that the puncture be mapped to $\xi_{j}=0$ and that at the puncture

$$
\frac{d \xi_{j}}{d z_{j}}\left(z_{j}=0\right)=1
$$

Under this condition, the radius $r_{j}$, called the mapping radius of $D_{j}$, is uniquely determined. The $\xi_{j}$ coordinate is called the natural coordinate. The reduced 


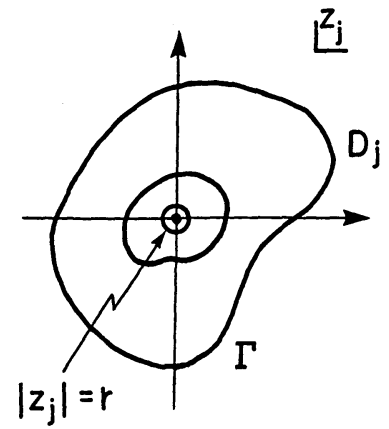

(a)

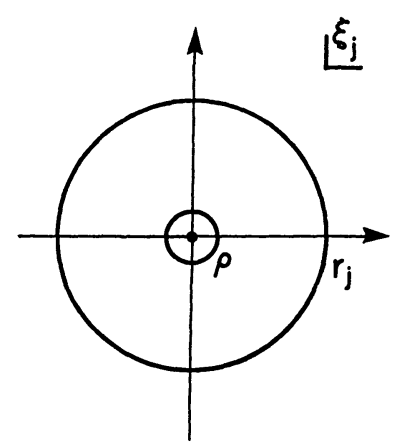

(b)

Fig. 2. a A punctured disk $D_{j}$ given by the region of the $z_{j}$-plane enclosed by the curve $\Gamma$. The puncture is at $z_{j}=0$. b The punctured disk is mapped into a circular disk of radius $r_{j}$. The coordinate $\xi_{j}$ is called the natural coordinate for the punctured disk. The puncture is at $\xi_{j}=0$, and $d \xi_{j} / d z_{j}=1$ at the puncture

modulus $M_{j}$ of the disk $D_{j}$ is defined to be

$$
M_{j}=\frac{1}{2 \pi} \ln r_{j}
$$

It is useful to obtain the reduced modulus $M_{j}$ by a limiting procedure. Consider the circle $\left|z_{j}\right|=r$ and define $M_{j}(r)$ to be the modulus of the annular domain $D_{j}(r)$ bounded by this circle and the curve $\Gamma$. Since near the puncture $\xi_{j}$ and $z_{j}$ are related by Eq. (2.2.5) one has:

$$
\xi_{j}=z_{j}+a z_{j}^{2}+\ldots \rightarrow \frac{\xi_{j}}{z_{j}}=1+a z_{j}+\ldots
$$

The image of the circle $\left|z_{j}\right|=r$ in the $\xi_{j}$-plane is a closed curve with $\varrho_{<}=\min _{\left|z_{j}\right|=r}\left|\xi_{j}\right|$ being the shortest distance to the origin, and $\varrho_{>}=\max _{\left|z_{j}\right|=r}\left|\xi_{j}\right|$ being the largest distance to the origin. It follows from Eq. (2.2.7) that

$$
\frac{\left|\xi_{j}\right|}{r}=\left|1+a z_{j}+\ldots\right|=1+\mathcal{O}(r)
$$

and therefore

$$
\lim _{r \rightarrow 0} \frac{\varrho_{<}}{r}=\lim _{r \rightarrow 0} \frac{\varrho_{>}}{r}=1
$$

The image of the domain $D_{j}(r)$ in the $\xi_{j}$ plane is an annular region whose outer boundary is $\left|\xi_{j}\right|=r_{j}$ and whose inner boundary lies in the region $\varrho_{<} \leqq\left|\xi_{j}\right| \leqq \varrho_{>}$. It therefore follows that the modulus $M_{j}(r)$, because of monotonicity must satisfy the 
inequalities:

$$
\frac{1}{2 \pi} \ln \frac{r_{j}}{\varrho_{>}} \leqq M_{j}(r) \leqq \frac{1}{2 \pi} \ln \frac{r_{j}}{\varrho_{<}}
$$

Adding to each term the quantity $\frac{1}{2 \pi} \ln r$ one finds

$$
\frac{1}{2 \pi} \ln \frac{r}{\varrho_{>}}+\frac{1}{2 \pi} \ln r_{j} \leqq M_{j}(r)+\frac{1}{2 \pi} \ln r \leqq \frac{1}{2 \pi} \ln \frac{r}{\varrho_{<}}+\frac{1}{2 \pi} \ln r_{j},
$$

and finally taking the limit as $r \rightarrow 0$, making use of Eq. (2.2.9) one finds

$$
\lim _{r \rightarrow 0}\left(M_{j}(r)+\frac{1}{2 \pi} \ln r\right)=\frac{1}{2 \pi} \ln r_{j}=M_{j} .
$$

Thus the reduced modulus is just the limit of the ordinary modulus of the annulus $D_{j}(r)$ as the radius $r$ goes to zero, with the leading divergence subtracted away.

Let us denote by $D_{j}(\varrho)$ the annulus $\varrho \leqq\left|\xi_{j}\right| \leqq r_{j}$ in the $\xi_{j}$-plane (see Fig. 2). Its modulus, denoted by $M_{j}(\varrho)$ is given by $M_{j}(\varrho)=\frac{1}{2 \pi} \ln \left(r_{j} / \varrho\right)$. Require now that curves homotopic to the puncture in this annulus have lengths greater than $a$. The JSquadratic differential

$$
\phi\left(\xi_{j}\right)=-\frac{a^{2}}{(2 \pi)^{2}} \frac{1}{\xi_{j}^{2}}
$$

gives rise to a metric $|\phi|^{1 / 2}|d z|$ which is admissible for the constant $a$. It follows from our previous discussion that

$$
\|\phi\|_{D_{j}(\varrho)}=\iint_{D_{j}(\varrho)}|\phi| d^{2} \xi_{j}=a^{2} M_{j}(\varrho) \leqq \iint_{D_{j}(\varrho)} \varrho^{2} d^{2} \xi_{j},
$$

for any metric $\varrho$ admissible for the constant $a$.

We now want to show that the reduced area associated with the metric arising from the JS differential is just given by the reduced norm, $a^{2} M_{j}$, of the quadratic differential (here $M_{j}$ is the reduced modulus). Consider again the annulus $D_{j}(r)$, it follows that

$$
\iint_{D_{j}(\varrho>)}|\phi| d^{2} \xi_{j} \leqq \iint_{D_{j}(r)}|\phi| d^{2} z_{j} \leqq \iint_{D_{j}\left(\varrho_{<}\right)}|\phi| d^{2} \xi_{j} .
$$

Using the explicit expression in Eq. (2.2.13) one finds

$$
\frac{a^{2}}{2 \pi} \ln \frac{r_{j}}{\varrho_{>}} \leqq \iint_{D_{j}(r)}|\phi| d^{2} z_{j} \leqq \frac{a^{2}}{2 \pi} \ln \frac{r_{j}}{\varrho_{<}} .
$$

Adding $\frac{a^{2}}{2 \pi} \ln r$ to each term one has

$$
\frac{a^{2}}{2 \pi} \ln r_{j}+\frac{a^{2}}{2 \pi} \ln \frac{r}{\varrho_{>}} \leqq \int_{D_{j}(r)}|\phi| d^{2} z_{j}+\frac{a^{2}}{2 \pi} \ln r \leqq \frac{a^{2}}{2 \pi} \ln r_{j}+\frac{a^{2}}{2 \pi} \ln \frac{r}{\varrho_{<}},
$$

and taking the limit as $r \rightarrow 0$ one gets, using Eq. (2.2.9),

$$
\lim _{r \rightarrow 0}\left(\iint_{D_{j}(r)}|\phi| d^{2} \xi+\frac{a^{2}}{2 \pi} \ln r\right) \equiv \mathscr{A}\left(|\phi|^{1 / 2}\right)=a^{2} M_{j},
$$


showing, as we wanted, that the reduced area for the $\phi$-metric equals the reduced norm $a^{2} M_{j}$, in complete analogy with the case for regular annuli [in Eq. (2.2.3)].

The next relevant property is that for any metric $\varrho$ defined on the punctured disk and admissible for the constant $a$, the associated reduced area $\mathscr{A}(\varrho)$ will be greater or equal to the reduced area $\mathscr{A}\left(|\phi|^{1 / 2}\right)$ associated with the JS quadratic differential with closed horizontal trajectories of length $a$. Begin with the relation $a^{2} M_{j}(\varrho) \leqq \iint_{D_{j}(\varrho)} \varrho^{2} d^{2} \xi_{j}$, in Eq. (2.2.14). For a given $\varrho$ the circle $\left|\xi_{j}\right|=\varrho$ corresponds, in the $z_{j}$-plane, to a closed curve with $r_{<}=\min _{\left|\xi_{j}\right|=\varrho}\left|z_{j}\right|$ being the shortest distance to the
origin. It follows that

$$
a^{2} M_{j}(\varrho) \leqq \int_{D_{j}(r<)} \varrho^{2} d^{2} z_{j}
$$

Adding $\frac{a^{2}}{2 \pi} \ln r_{<}$to both sides of the inequality one finds

$$
\frac{a^{2}}{2 \pi} \ln r_{j}+\frac{a^{2}}{2 \pi} \ln \frac{r_{<}}{\varrho} \leqq \int_{D_{j}\left(r_{<}\right)} \varrho^{2} d^{2} z_{j}+\frac{a^{2}}{2 \pi} \ln r_{<} .
$$

Taking the limit as $\varrho \rightarrow 0$, using $\lim _{\varrho \rightarrow 0}\left(r_{<} / \varrho\right)=1$ (as in Eq. (2.2.9)) one finds that

$$
a^{2} M_{j} \leqq \lim _{r<\rightarrow 0}\left(\iint_{D_{j}\left(r_{<}\right)} \varrho^{2} d^{2} \xi_{j}+\frac{a^{2}}{2 \pi} \ln r_{<}\right)=\mathscr{A}(\varrho),
$$

which was the desired result. Summarizing; the results in Eqs. (2.2.18) and (2.2.21) imply that

$$
\mathscr{A}\left(|\phi|^{1 / 2}\right)=a^{2} M_{j} \leqq \mathscr{A}(\varrho),
$$

where $\phi$ is a quadratic differential with closed horizontal trajectories of length $a$ in a punctured disk of reduced modulus $M_{j}$, and $\varrho$ is any metric on the punctured disk admissible for the constant $a$.

This section is concluded by explaining a composition property for moduli and for reduced moduli. Consider three non-intersecting simple closed Jordan curves $\gamma_{a}, \gamma_{b}$, and $\gamma_{c}$, with $\gamma_{a}$ enclosing $\gamma_{b}$, and $\gamma_{b}$ enclosing $\gamma_{c}$ as illustrated in Fig. 3a. Denote by $M_{i j}$ the modulus of the annular domain bounded by the curves $\gamma_{i}$ and $\gamma_{j}$. It can be shown $[\mathrm{Ah}]$ that

$$
M_{a c} \geqq M_{a b}+M_{b c} .
$$

A similar relation holds for reduced modulus. Consider the punctured disk $D_{a}$ bounded by the curve $\gamma_{a}$, and the punctured disk $D_{b}$ bounded by the curve $\gamma_{b}$, enclosed by $\gamma_{a}$ as illustrated in Fig. 3b. Let the puncture be at $z=0$. Consider the auxiliary curve $\gamma_{c}$ defined by $|z|=r$. It follows from Eq. (2.2.23) that

$$
M_{a c}+\frac{1}{2 \pi} \ln r \geqq M_{a b}+\left(M_{b c}+\frac{1}{2 \pi} \ln r\right),
$$

and taking the limit as $r \rightarrow 0$ one finds

$$
M_{a} \geqq M_{a b}+M_{b},
$$




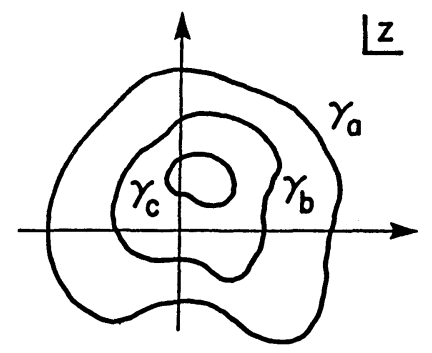

(a)

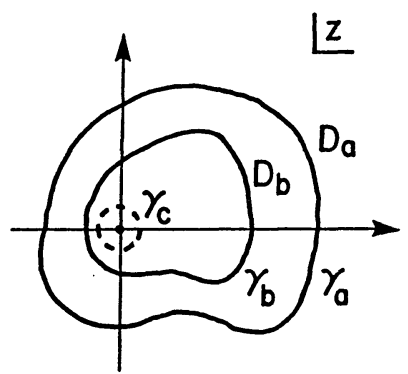

(b)

Fig. 3. a If $M_{i j}$ denotes the modulus of the annulus enclosed by $\gamma_{i}$ ands $\gamma_{j}$, one has, for the above figure that $M_{a c} \geqq M_{a b}+M_{b c}$. b For punctured disks a similar relation holds: $M_{a} \geqq M_{a b}+M_{b}$, where $M_{i}$ denotes the reduced modulus of the disk bounded by $\gamma_{i}$. The dotted curve $\gamma_{c}$ is an auxiliary curve

where $M_{a}$ and $M_{b}$ denote the reduced moduli of $D_{a}$ and $D_{b}$ respectively, and use was made of Eq. (2.2.12).

\subsection{Admissible Curves and Feynman Graphs}

An admissible system of curves on a punctured Riemann surface is a set of nonintersecting simple closed Jordan curves. The curves must not be homotopic to each other nor to the punctures. The maximal number of such curves in an admissible system is finite. For a genus $g, n$-punctured surface this number is equal to $3(g-1)+n$ (see, for example [St 1]).

Consider, for example, the case of a four-punctured sphere. Here an admissible set of curves may have no curves, or one curve. If there is a curve, it must separate two punctures from the other two. It is clear that one cannot add an extra curve, because any additional nonintersecting curve would either be homotopic to the original one, or homotopic to a puncture. There are three ways of separating the punctures, corresponding to the $s, t$, and $u$-diagrams of field theory. This is not to say that there are just three inequivalent sets of admissible curves. Suppose a curve separates punctures 1 and 2 from punctures 3 and 4 . One can perform a Dehn twist across an annulus homotopic to a curve separating punctures 1 and 3 from 2 and 4 . The final curve still separates the same punctures, thus the corresponding Feynman graph is the same. The final curve, however, is not homotopic to the original curve. The result is that via Dehn twists, which are generators of modular transformations, a given Feynman graph corresponds to an infinite number of different admissible curves. In other words, the Feynman graphs are in direct correspondance with the possible admissible sets of curves divided by the action of modular transformations.

While it has been well known that to a maximal set of admissible curves one can associate a $\phi^{3}$ Feynman graph, if we consider all possible sets of admissible curves on a surface we obtain the Feynman diagrams of a completely nonpolynomial scalar field theory. This is in fact true even for loops; once we consider all possible sets of curves we find the Feynman graphs of a field theory that includes quantum vertices of all genus, and with all numbers of punctures. 


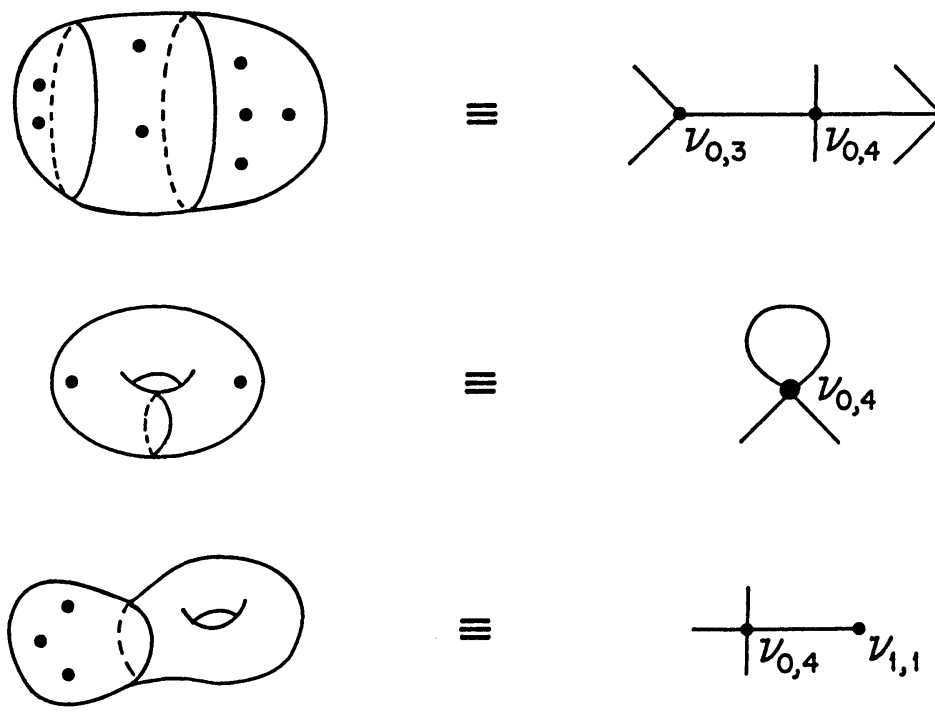

Fig. 4. To every closed Riemann surface with punctures and an admissible set of curves one can associate a Feynman graph. Vertices are denoted by $\mathscr{V}_{g, n}$, where $g$ counts the number of loops it carries, and $n$ denotes the number of legs

Given a set of admissible curves on a punctured Riemann surface $R$ the corresponding Feynman graph is deduced as follows. The surface $R$ is cut along the admissible curves. One gets a number of surfaces $\left\{R_{i}\right\}$, each one with some number of holes whose boundaries are the admissible curves, and a number of punctures. To each surface $R_{i}$ with $p_{i}$ boundaries and $m_{i}$ punctures we associate a contact interaction with $n_{i}=\left(p_{i}+m_{i}\right)$ lines, out of which $p_{i}$ are to be internal, and the remaining $m_{i}$ are external. For each surface $R_{i}$ one can compute the genus $g_{i}$ of the surface obtained by shrinking away the boundaries and deleting the punctures. If $g_{i}=0$, the surface $R_{i}$ corresponds to a contact interaction of the classical action, it is a classical vertex denoted as $\mathscr{V}_{0, n_{i}}$. If $g_{i} \neq 0$, the surface corresponds to a contact interaction of the quantum action (proportional to $\left.(\hbar)^{g}\right)$, it is a quantum vertex denoted as $\mathscr{V}_{g_{i}, n_{i}}$. This is done for all surfaces $\left\{R_{i}\right\}$. The pair of lines arising from each admissible curve must be joined; these are the internal lines. The result is the Feynman graph associated with the admissible set in $R$. Three examples are given in Fig. 4.

There is also a simple correspondence between JS-quadratic differentials and Feynman graphs. The characteristic ring domains of the quadratic differential consist of annuli and punctured disks; out of each annulus pick a closed trajectory $\gamma_{i}$. The set of $\gamma_{i}$ 's is the admissible set of curves corresponding to the quadratic differential, and from it the Feynman graph is constructed using the prescription given above. It follows that the maximum number of annuli equals $(3 g-3+n)$, namely, the maximum number of curves in an admissible set. It turns out that each punctured disk corresponds to an external line, each internal annulus corresponds to an internal propagator line, and the critical graphs (whose edges are critical trajectories joining zeroes of the quadratic differential) correspond to the interaction vertices. 


\section{Closed String Field Theory and its Quadratic Differentials}

\subsection{The Polyhedra of Closed String Field Theory}

In this section the main features of the construction of classical closed string theory presented in $[\mathrm{SaZw}]$ and $[\mathrm{KKS}]$ will be briefly reviewed. It will be possible to streamline somewhat the ideas. A somewhat novel point is that it turns out that the trivalence condition on critical graphs need not be imposed explicitly as a condition on restricted polyhedra.

Classical closed string theory was constructed using as a basic tool the following theorem by Strebel:

Theorem 3.1 (Theorem 23.5 in [St 1]). For any n-punctured Riemann surface ( $n \geqq 1$, except for $g=0$, where $n \geqq 3)^{4}$ there is a unique JS-quadratic differential whose characteristic ring domains are $n$ punctured disks of specified circumferences $A_{j}$.

It is convenient to define the notion of the polyhedron corresponding to a surface $[\mathrm{SaZw}]$.

Definition. The polyhedron of a punctured Riemann surface is the critical graph of the quadratic differential of Theorem 3.1, when all $A_{j}$ 's are chosen equal to $2 \pi$.

The faces of the polyhedron are the closed critical trajectories homotopic to the closed horizontal trajectories in every punctured disk. It follows that the perimeter of each face is equal to $2 \pi$. The polyhedron is completely specified by the topology of the graph, the labeling of the faces, and the lengths of all edges (measured with the $\phi$-metric).

It is also useful to define the notion of a restricted polyhedron.

Definition. A restricted polyhedron is a polyhedron in which any nontrivial closed edge-path has length greater or equal to $2 \pi$.

We shall also call the $(n \geqq 3, g=0)$-polyhedra, classical polyhedra ${ }^{5}$ and the $(n \geqq 1, g \geqq 1$ ) polyhedra, quantum polyhedra (the same terminology will apply to restricted polyhedra).

It was argued in $[\mathrm{SaZw}]$, using Theorem 3.1, that any $n$-punctured Riemann surface could be built as a contact interaction by gluing the edges of $n$-semiinfinite cylinders of circumferences $2 \pi$ across the faces of a unique polyhedron. The polyhedron has some number $e$ of edges, whose lengths play the role of modular parameters. Given the $n$ face perimeter constraints, the number of unconstrained parameters is $(e-n)$. From the Euler theorem for polyhedra we have that

$$
n-e+v=2-2 g,
$$

where $v$ denotes the number of vertices. A simple rearrangement of this relation gives

$$
(e-n)=(6 g-6+2 n)-(2 e-3 v) \text {. }
$$

4 The theorem was stated in [St 1] as holding for $n \geqq 2$, however, other theorems in [St 1] guarantee that it actually holds for $n=1$. The reader may consult [Ha], or the detailed discussion of [SoZw 2]

5 One may wish to call a circle of circumference $2 \pi$ a two-faced $(n=2)$ restricted polyhedron. To this polyhedron one can attach two semiinfinite cylinders giving rise to a two-punctured sphere. This polyhedron is not a critical trajectory, it is just a horizontal trajectory 
The first term in the right-hand side, $(6 g-6+2 n)$ is the real dimension $\operatorname{dim}\left(\mathscr{M}_{g, n}\right)$ of the moduli space of the genus $g, n$-punctured surfaces. In order that the number of unconstrained parameters $(e-n)$ in the polyhedron equal $\operatorname{dim}\left(\mathscr{M}_{g, n}\right)$ one requires that $2 e=3 v$, which implies that all vertices in the polyhedron are trivalent. This condition was imposed explicitly in $[\mathrm{SaZw}]$. It will be seen now that the cases when there are higher valence vertices need not be ruled out explicitly since they correspond to polyhedra with a lower number of unconstrained parameters. Denote by $v_{k}$ the number of vertices in the polyhedron with valence $k$. It then follows that for any polyhedron one has $v=\sum_{k} v_{k}$, and

$$
2 e=3 v_{3}+\sum_{k=4} k v_{k} \text {. }
$$

Using the above, Eq. (3.1.2) can be rewritten as

$$
(e-n)=\operatorname{dim}\left(\mathscr{M}_{g, n}\right)-\sum_{k=4}(k-3) v_{k},
$$

showing that if there are vertices of valence greater than three the number of unconstrained parameters in the polyhedron goes below that necessary to describe the corresponding moduli space. It does no harm, therefore, to include along with the trivalent polyhedra all other polyhedra with higher valence vertices. In fact, it may be necessary to include them to ensure continuity. As some edge on a polyhedron becomes of zero length two vertices of valence three coalesce to form a vertex of valence four; this is typically a crossover point into another polyhedron of different topology. It seems fairly unnatural to remove those special points since it would ruin the continuity among the various polyhedra.

Since in field theory some of the surfaces arise by Feynman graphs with internal propagators, and some arise by contact interactions, not all surfaces should be built using the corresponding polyhedron. After some preliminary results $[\mathrm{SaZw}]$ the complete condition that determines the polyhedra required for the classical field theory vertices (genus zero $n \geqq 3$ punctures) was arrived at in [KKS]. The vertices of the classical field theory are the restricted classical polyhedra. Polyhedra, as explained in [SoZw 2], lead to interaction vertices that satisfy the requisite properties of permutation symmetry between scattering strings and of hermiticity.

The Feynman graphs of the field theory are constructed by joining restricted classical polyhedra with propagators, namely, tubes of perimeter $2 \pi$. These Feynman graphs correspond to JS-quadratic differentials with second order poles at the punctures. All residues are identical and such that closed horizontal trajectories homotopic to the punctures have lengths $2 \pi$. The characteristic ring domains are punctured disks and annuli, the latter, correspond to the propagators and also have closed horizontal trajectories of length $2 \pi$. For any $n$-string tree amplitude ( $n$-punctured Riemann sphere) the number of intermediate annuli or propagators can go from zero, for the pure $n$-vertex, up to $(n-3)$, which is the maximum number of curves in an admissible set (as discussed in Sect. 2.3) and corresponds to the case when the Feynman graph is built exclusively out of three point vertices.

There is a property of restricted polyhedra that will be of utility later. Given two restricted polyhedra, pick one face in each of them, glue them across these faces (the gluing must respect lengths!). The result is another restricted polyhedron. The reader may consult the simple proof given in [KKS]. The result, in fact, holds both for classical or quantum restricted polyhedra. 


\subsection{Proposed Existence and Uniqueness Theorem}

In this section an existence and uniqueness theorem for quadratic differentials will be proposed. Then it will be shown that as a corollary, it guarantees the correct covering (without overcounting or undercounting) of the moduli spaces of punctured Riemann spheres by the tree amplitudes of covariant closed string field theory. The proof of this existence and uniqueness theorem will be given in Sect. 7 .

Theorem 3.2. Given a Riemann sphere with $n \geqq 3$ distinguishable punctures there is $a$ unique JS quadratic differential satisfying the following conditions:

(i) The singularities are $n$ second order poles at the punctures,

(ii) The characteristic ring domains are $n$ punctured disks and $k$ annuli, where $0 \leqq k$ $\leqq n-3$,

(iii) The lengths of the closed horizontal trajectories on the punctured disks and annuli are all equal to $2 \pi$,

(iv) Every nontrivial closed critical trajectory has length (measured using the quadratic differential) greater or equal to $2 \pi$.

Let us show that the above theorem guarantees the correctness of classical closed string theory. Denote the set of all quadratic differentials satisfying the above properties (for a fixed $n$ ) by $S_{Q}$. Denote the set of quadratic differentials constructed by the tree level Feynman graphs by $S_{F}$. It is clear from the Feynman rules, which use tubes of perimeter $2 \pi$ for the propagators and restricted classical polyhedra for the vertices, that any Feynman graph defines a quadratic differential that satisfies the above four properties. In fact, condition (iv) corresponds to the requirement that the polyhedra must be restricted polyhedra. Therefore, $S_{F} \subseteq S_{Q}$. Moreover, any quadratic differential from $S_{Q}$ can be obtained from some Feynman graph, since if we isolate the various disconnected critical graphs, they correspond (because of (iv)) to restricted classical polyhedra. Therefore $S_{Q} \subseteq S_{F}$. It follows that $S_{F}=S_{Q}$, that is, the set of all Feynman graphs for $n$-string scattering construct all the quadratic differentials satisfying the above properties. Consider now the map from $S_{Q}$ to the space of $n$-punctured, genus zero Riemann surfaces $S_{R}$. The map is one to one; two different quadratic differentials cannot correspond to the same Riemann surface because this would violate the uniqueness aspect of Theorem 3.2. The map is also onto; any Riemann surface arises from some quadratic differential in the set, because of the existence aspect of Theorem 3.2. It follows that the map is an isomorphism between sets and $S_{Q}=S_{R}$. Using our previous result $S_{F}=S_{Q}$, it follows that $S_{F}=S_{R}$. In summary, it has been shown that:

Corollary. The Feynman graphs of classical closed string theory generate the moduli space $\mathscr{M}_{0, n}$ of the n-punctured Riemann spheres.

For any $n \geqq 3$ punctured sphere one has a finite number of distinct Feynman graphs (four for $n=4$, twenty six for $n=5$, and so on). These Feynman graphs cover once and only once $\mathscr{M}_{0, n}$, thus they generate a cell decomposition of $\mathscr{M}_{0, n}$. Each Feynman graph gives a cell.

\section{Extremal Properties of Quadratic Differentials}

In this section we explore the extremal properties of quadratic differentials. We will review results of Jenkins concerning holomorphic quadratic differentials with 
specified circumferences. Here the characteristic ring domains are annuli. What we need for closed string theory is a theorem for quadratic differentials whose characteristic ring domains are punctured disks and intermediate annuli. While the theorem of Jenkins considers the case of no punctured disks, most of the results of Strebel consider the case where there are only punctured disks. The results of Strebel useful to us are also reviewed and some of them recast as extremal problems.

Let us begin with an existence and uniqueness theorem formulated by Jenkins for holomorphic quadratic differentials on an arbitrary Riemann surface.

Theorem 4.1 (Jenkins [Je]). Consider an admissible set of curves $\left(\gamma_{i}\right)$ on a Riemann surface and an associated set of constants $A_{i}$.

(a) There is a unique JS quadratic differential whose characteristic ring domains are annuli (some may be degenerate) homotopic to the curves $\gamma_{i}$ such that for the rings that do not collapse $\left(M_{i}>0\right)$ the lengths $a_{i}$ of the closed horizontal trajectories homotopic to $\gamma_{i}$ satisfy: $a_{i}=A_{i}$, and for the collapsed rings $\left(M_{i}=0\right)$ the minimal length $a_{i}$ among all closed curves homotopic to $\gamma_{i}$ exceeds or equals $A_{i}$, namely: $a_{i} \geqq A_{i}$.

(b) The above quadratic differential is the unique solution to the problem of maximizing the functional

$$
\mathscr{F}=\sum_{i} A_{i}^{2} M_{i}
$$

among all possible choices of disjoint ring domains $R_{i}$ homotopic to $\gamma_{i}$, where $M_{i}$ is the modulus of $R_{i}$. The characteristic ring domains of the quadratic differential provide the set of ring domains of maximal $\mathscr{F}$. The maximal possible value of $\mathscr{F}$ is denoted as $\mathscr{F}(\phi)$. (For the case $g=1, n=0$ the maximizing domain is not unique).

It is interesting to note that one cannot quite specify the circumferences of the quadratic differential, some may be realized, if their corresponding rings do not collapse. For the collapsed rings we known that the circumferences are greater or equal to the specified parameters. This is just what happens in covariant closed string theory; propagators are required to have a circumference of $2 \pi$, but when they collapse the length of homotopic closed trajectories grows, as discussed in [Sa]. ${ }^{6}$ Using Eq. (2.2.3) it follows that for each ring domain that does not collapse $A_{i}^{2} M_{i}=\int|\phi| d x d y$, where $A_{i}$ is the length of the closed trajectories in the ring. Adding up the contributions of all the rings one has

$$
\mathscr{F}(\phi)=\iint_{R}|\phi| d x d y \equiv\|\phi\|_{R},
$$

that is, the extremal value of $\mathscr{F}$ is the area of the surface computed with the metric associated with the quadratic differential.

The above quadratic differential solves another related extremal problem [Je]. Consider the set of admissible curves $\gamma_{i}$ of Theorem 4.1, and the associated set of numbers $A_{i}$. Note that the above quadratic differential $\phi$ gives rise to a metric admissible for the $A_{i}$ 's, since the lengths of closed trajectories homotopic to the curves $\gamma_{i}$ satisfy the appropriate length conditions. Let the metric $\varrho(z)|d z|$ be admissible for the $A_{i}$ 's. It follows directly from Eq. (2.2.3) applied to each ring domain that

$$
\mathscr{F}(\phi)=\|\phi\|_{R} \leqq \iint_{R} \varrho^{2} d x d y,
$$

${ }^{6}$ I thank M. Saadi for bringing to my attention this property of the Jenkins construction 
namely, the area measured with any admissible metric exceeds or equals that measured with the quadratic differential of Theorem 4.1. Thus, the metric that gives the lowest possible area among all possible admissible metrics, called the extremal metric, is that associated with the quadratic differential of Theorem 4.1. This metric is unique.

Let us now consider some results of Strebel:

Theorem 4.2 (Theorem 23.1 in [St 1]). Consider a JS-quadratic differential whose characteristic ring domains are a finite number of punctured discs $R_{j}$, with punctures $P_{j}$ and reduced moduli $M_{j}$ (with respect to a fixed system of coordinates at the punctures); and a finite number of ring domains $R_{k}$ with finite moduli $M_{k} \geqq 0$. Let $a_{j}$ denote the length of the closed horizontal trajectories surrounding the punctures $P_{j}$ (no punctured disk can be degenerate). Let $a_{k}$ denote, for rings with $M_{k}>0$, the length of the closed horizontal trajectories homotopic to $\gamma_{k}$, and for rings with $M_{k}=0$, the minimal length among all curves homotopic to $\gamma_{k}$.

Consider the reduced norm of the quadratic differential

$$
\mathscr{F}=\sum_{j} a_{j}^{2} M_{j}+\sum_{k} a_{k}^{2} M_{k} .
$$

It follows that for any other competing set of domains $\widetilde{R}_{j}, \widetilde{R}_{k}$ of the homotopy type of $\gamma_{j}, \gamma_{k}$, and with moduli $\tilde{M}_{j}, \tilde{M}_{k}$ one has that

$$
\sum_{j} a_{j}^{2} \tilde{M}_{j}+\sum_{k} a_{k}^{2} \tilde{M}_{k} \leqq \mathscr{F} .
$$

The equality holds only if all domains are identical: $\widetilde{R}_{j}=R_{j}, \widetilde{R}_{k}=R_{k}$.

(The conventions of [St 1] are followed, annuli are denoted by the letter $k$, punctured disks by the letter $j$, and both by the letter $i$.) Our main objective in Sect. 5 will be to address the issue of existence of such quadratic differentials. Strebel does show the existence and uniqueness of a quadratic differential with closed trajectories whose characteristic ring domains are just punctured disks (this is Theorem 3.1 reviewed in the previous section). It follows quite simply from Theorems 3.1, 4.2, and our discussion in Sect. 2.2 that the quadratic differentials with only punctured disks solve the following extremal problems:

Theorem 4.3. Consider an $n$-punctured ( $n \geqq 3$ for $g=0$, and $n \geqq 1$ for $g \geqq 1$ ) Riemann surface, and consider $n$ disjoint punctured disks $R_{j}$ around the punctures with reduced moduli $M_{j}$ with respect to some fixed coordinates at the punctures. The following quantity

$$
\mathscr{F}=\sum A_{j}^{2} M_{j},
$$

will be maximized by a unique choice of punctured disks $\bar{R}_{j}$ with reduced moduli $\bar{M}_{j}$. The $\bar{R}_{j}$ 's are the characteristic domains of a uniquely determined ${ }^{7}$ quadratic differential $\phi$ with second order poles at the punctures and with closed horizontal trajectories of lengths $A_{j}$. Moreover,

$$
\mathscr{F}(\phi)=\sum A_{j}^{2} \bar{M}_{j}=\mathscr{A}\left(|\phi|^{1 / 2}\right) \leqq \mathscr{A}(\varrho),
$$

namely, the maximal value of $\mathscr{F}$ equals the reduced area of the quadratic differential and it is the lowest possible value for the reduced area of any metric on the surface admissible for the given $A_{j}$ 's.

\footnotetext{
7 The quadratic differential is, of course, independent of the choice of local coordinate at the punctures. This will always be the case in the latter theorems too
} 
Proof. The correctness of the above is argued as follows. Because of Theorem 3.1, there is a unique quadratic differential second order poles and with closed trajectories of lengths $A_{i}$. Given that the quadratic differential exist, it follows from Theorem 4.2 that it must maximize $\mathscr{F}$, which is just what the functional in Eq. (4.4) reduces to when there are no intermediate annuli. Finally, it follows from Eq. (2.2.22), used for all punctured disks, that Eq. (4.7) holds.

With this result we can reinterpret the Witten vertex as a solution of the following extremal problem: Given a three punctured sphere, with some arbitrary (but fixed) coordinate systems around the punctures, find the set of nonoverlapping punctured disks that maximizes the sum of reduced moduli $\left(M_{1}+M_{2}+M_{3}\right)$. Answer: the characteristic ring domains of the unique (up to scale) quadratic differential with equal residues at the punctures. This is the Witten vertex, as explained in detail in [SoZw 2]. Similarly, the light-cone three point vertex coupling strings of lengths $l_{1}, l_{2}$, and $l_{3}=l_{1}+l_{2}$, is the solution of extremizing on a three punctured sphere the functional $\left(l_{1}^{2} M_{1}+l_{2}^{2} M_{2}+l_{3}^{2} M_{3}\right)$. Both quadratic differentials give rise to the metrics with lowest possible reduced area given the appropriate length conditions for curves surrounding the punctures.

\section{Quadratic Differentials with Punctured Disks and Annuli}

In this section a new existence and uniqueness theorem for quadratic differentials with second order poles is established. This theorem establishes that given a Riemann surface with some punctures and a homotopy class of admissible curves $\left(\gamma_{i}\right)$, one can specify residues of second order poles at the punctures and lengths of closed trajectories homotopic to the $\gamma_{i}$ (in the sense described below). This result is obtained using the theorems of Jenkins and Strebel reviewed in the previous section.

Theorem 5.1. Consider a genus $g$ Riemann surface with $n \geqq 0$ punctures ( $n \geqq 3$ for $g=0)$ with an admissible set of curves $\gamma_{k}$ and $n$ curves $\gamma_{j}$ surrounding the punctures. Let $A_{k}$ denote a set of constants associated with the curves $\gamma_{k}$, and $A_{j}$ denote a set of constants associated with the curves $\gamma_{j}$.

(a) There is a unique JS quadratic differential whose characteristic ring domains include $n$ non-degenerate punctured disks with closed horizontal trajectories of lengths $A_{j}$ and annuli (some may be degenerate) homotopic to the curves $\gamma_{k}$. For the rings that do not collapse $\left(M_{k}>0\right)$ the lengths $a_{k}$ of the closed horizontal trajectories homotopic to $\gamma_{k}$ must satisfy: $a_{k}=A_{k}$, and for the collapsed rings $\left(M_{k}=0\right)$ the minimal length $a_{k}$ among all closed curves homotopic to $\gamma_{k}$ must exceed or equal $A_{k}$, namely: $a_{k} \geqq A_{k}$.

(b) The above quadratic differential is the unique solution to the problem of maximizing the functional

$$
\mathscr{F}=\sum A_{j}^{2} M_{j}+\sum A_{k}^{2} M_{k},
$$

among all possible choices of disjoint ring domains $R_{j}, R_{k}$ homotopic to $\gamma_{j} \gamma_{k}$, where $M_{j}$ is the reduced modulus with respect to a fixed set of local coordinates at the punctures, and $M_{k}$ is the modulus of $R_{k}$. The characteristic ring domains of the quadratic differential provide the set of ring domains of maximal $\mathscr{F}$. The maximal possible value of $\mathscr{F}$ is denoted as $\mathscr{F}(\phi)$. (The maximizing domain is not unique for the case $g=1, n=0$.) 
Proof. Let us first deal with the uniqueness of the quadratic differential (part (a)). The following is a simple extension of the proof presented in [St 1] for the uniqueness in Theorem 4.1. Let $\phi$ and $\tilde{\phi}$ denote two different quadratic differentials satisfying the conditions of part (a). Namely, $a_{j}=\tilde{a}_{j}=A_{j}$ for the punctured disks, and $a_{k}=A_{k}$ if $M_{k}>0, a_{k} \geqq A_{k}$ if $M_{k}=0$, and similarly $\tilde{a}_{k}=A_{k}$ if $\tilde{M}_{k}>0, \tilde{a}_{k} \geqq A_{k}$ if $\tilde{M}_{k}=0$. It then follows using Theorem 4.2 , with $\mathscr{F}$ defined by the quadratic differential $\phi$ that

$$
\sum a_{i}^{2} M_{i} \geqq \sum a_{i}^{2} \tilde{M}_{i} \geqq \sum A_{i}^{2} \tilde{M}_{i}=\sum \tilde{a}_{i}^{2} \tilde{M}_{i},
$$

where in the first inequality we have used Theorem 4.2, in the second inequality we have used $a_{i} \geqq A_{i}$, and for the equality we have used the fact that for $\tilde{M}_{k} \neq 0, \tilde{a}_{k}=A_{k}$. Starting the other way around, using $\bar{\phi}$ instead of $\phi$, we would obtain the above inequality but in the other direction. It follows that all the inequalities in Eq. (5.2) are actually equalities and therefore

$$
\sum a_{i}^{2} M_{i}=\sum a_{i}^{2} \tilde{M}_{i} .
$$

Using now Theorem 4.2, it follows that the two quadratic differentials must have identical ring domains. Thus they can only differ by a positive constant. This constant can only be one since the lengths of closed trajectories are also the same.

Let us now address the existence part. For this we show that the maximization of $\mathscr{F}$ in part (b) [Eq. (5.1)] gives us the desired quadratic differential. For any compact Riemann surface the modulus $M_{i}$ of any annulus (or punctured disk) homotopic to $\gamma_{i}$ is bounded above. Therefore $\mathscr{F}$ is bounded above. It follows (by a normal family argument) that there should exist a set of punctured disks and ring domains that maximize $\mathscr{F}$. Moreover, no punctured disk can collapse, because this makes $M \rightarrow-\infty$ and as a consequence $\mathscr{F} \rightarrow-\infty$. We now show that the maximizing set of domains gives rise to a quadratic differential. The basic idea is to cut out little disks including the punctures and to consider the truncated surface.

Let $z_{i}$ denote a fixed set of coordinate systems around the punctures. Consider the maximizing set of domains and let $\xi_{j}$ denote the natural coordinates around each of the punctures. In such coordinates the punctured disks appear as circles of radii $r_{j}$ and we also have that $\frac{d \xi_{j}}{d z_{j}}(0)=1$. The $r_{j}$ are nothing else than the mapping radii of the punctured disks, and therefore the reduced moduli of the punctured disks are given by $M_{j}=\frac{1}{2 \pi} \ln r_{i}$. We now cut out disks of radii $\varrho$ out of every punctured disk. Namely we excise the regions $\left|\xi_{j}\right| \leqq \varrho$, with $\varrho$ chosen such that $\varrho<\operatorname{Min}\left\{r_{j}\right\}$. The truncated surface is denoted by $R(\varrho)$. Denote by $M_{j}(\varrho)$ the modulus of the annulus $R_{j}(\varrho)$ that is obtained after excision of the $\varrho$-disk from the punctured domain. Clearly, $M_{j}(\varrho)=\frac{1}{2 \pi} \ln \frac{r_{j}}{\varrho}$. We now claim that in the cut surface $R(\varrho)$ the ring domains $R_{j}(\varrho)$, and $R_{k}$ (which are left unchanged) maximize the functional

$$
\mathscr{F}(\varrho)=\sum A_{j}^{2} M_{j}(\varrho)+\sum A_{k}^{2} M_{k}
$$

over all possible choices of annuli with the $A$ 's kept constant. Assume this is not true. Then there is a competing system of annuli with moduli $\tilde{M}_{j}(\varrho), \tilde{M}_{k}$ such that

$$
\mathscr{F}(\varrho)<\sum A_{j}^{2} \tilde{M}_{j}(\varrho)+\sum A_{k}^{2} \tilde{M}_{k} .
$$


We now show by restoring the $\varrho$-disks to the surface, that this leads to a contradiction with the initial assumption that $\mathscr{F}$ had been maximized in the original punctured surface. From Eqs. (5.1), (5.4) and the relation $M_{j}=M_{j}(\varrho)$ $+\frac{1}{2 \pi} \ln \varrho$, one finds

$$
\mathscr{F}=\mathscr{F}(\varrho)+\frac{1}{2 \pi} \ln \varrho \sum A_{j}^{2}
$$

Adding $\frac{1}{2 \pi} \ln \varrho \sum A_{j}^{2}$ to both sides of Eq. (5.5) and using Eq. (5.6) one obtains

$$
\mathscr{F}<\sum A_{j}^{2}\left(\tilde{M}_{j}(\varrho)+\frac{1}{2 \pi} \ln \varrho\right)+\sum A_{k}^{2} \tilde{M}_{k} .
$$

Making use of the inequality in (2.2.25) one has

$$
\tilde{M}_{j}(\varrho)+\frac{1}{2 \pi} \ln \varrho \leqq \tilde{M}_{j},
$$

where $\tilde{M}_{j}$ denotes the reduced modulus of the new punctured disk once we restore the $\varrho$-disk. The last two relations imply that

$$
\mathscr{F}<\sum A_{j}^{2} \tilde{M}_{j}+\sum A_{k}^{2} \tilde{M}_{k},
$$

which contradicts the original assumption that $\mathscr{F}$ had been maximized on the punctured surface. Thus because of the maximality of $\mathscr{F}(\varrho)$, we have a quadratic differential $\phi_{\varrho}$ on the cut surface $R(\varrho)$ obeying the particular conditions of the Jenkins construction. We know, moreover, that for sufficiently small $\varrho$, the annuli that correspond to the punctured disks cannot have collapsed. The closed horizontal trajectories of $\phi_{\varrho}$ are actually independent of $\varrho$. This is so because the intermediate annuli are fixed and do not depend on $\varrho$; and on the annuli that correspond to the punctured disks the closed trajectories are the lines $\left|\xi_{j}\right|=$ const, again $\varrho$-independent. It follows that the quadratic differentials $\phi_{\varrho}$ are just restrictions on $R(\varrho)$ of a single quadratic differential $\phi$ on $R$. This is a quadratic differential whose characteristic ring domains are punctured disks and annuli. The punctured domains will have closed trajectories of lengths $A_{j}$ and the intermediate annuli will have closed trajectories of lengths $A_{k}$ for the annuli that do not collapse.

As was the case for holomorphic quadratic differentials, the above quadratic differential also solves a minimal area problem:

Theorem 5.2. Consider a genus $g$ Riemann surface with $n \geqq 0$ punctures ( $n \geqq 3$ for $g=0)$ with a set of admissible curves $\gamma_{k}$ and associated constants $A_{k}$. Consider also the constants $A_{j}$ associated to closed curves surrounding the punctures. For any metric $\varrho|d z|$ admissible for the $A_{k}$ 's and $A_{j}$ 's one has

$$
\mathscr{F}(\phi)=\mathscr{A}\left(|\phi|^{1 / 2}\right) \leqq \mathscr{A}(\varrho),
$$

namely, the reduced area of $\varrho$ is bounded below by the reduced area (or reduced norm) of the quadratic differential $\phi$ which solves the extremal problem of Theorem 5.1 (here $\mathscr{F}(\phi)$ denotes the value of the functional $\mathscr{F}$ in Eq. (5.1) for the quadratic differential).

This theorem is an immediate consequence of Theorem 5.1 and Eqs. (2.2.3) and (2.2.22), used for all the characteristic ring domains of the quadratic differential. It 
should be noted that Theorems 3.1, 4.1, 4.2, and 4.3 are particular cases of Theorems 5.1 and 5.2 derived in this section.

\section{Simple Examples Showing No Overcounting of Surfaces}

Some examples will be given now that help us understand the meaning of Theorem 5.1 and also give a qualitative understanding for the lack of overcounting of surfaces by different Feynman graphs.

Because of the uniqueness aspect of Strebel's Theorem 3.1, it follows that the Feynman graphs containing pure contact interactions (restricted polyhedra) cannot give any overcounting. Each different restricted polyhedron corresponds to a different surface.

The condition that pure interactions are given by restricted polyhedra implies that no Feynman graph containing intermediate propagators (of circumference $2 \pi$ ) can produce a surface that is described by a classical or quantum polyhedron. The argument goes as follows. Suppose we have a surface with a quadratic differential that has some internal propagators. The corresponding annuli are homotopic to an admissible set of curves that we denote as $\left\{\gamma_{k}\right\}$. This quadratic differential is compatible, in the sense of part (a) of Theorem 5.1, with the set of constants $A_{i}=2 \pi$ associated to the curves surrounding the punctures and to the curves $\left\{\gamma_{k}\right\}$. Now assume that the surface can also be represented as a restricted polyhedron. It follows that all of the curves homotopic to the $\left\{\gamma_{k}\right\}$ in the polyhedron must have lengths greater than or equal to $2 \pi$. Then the quadratic differential associated with this restricted polyhedron is also compatible with the above set of constants (given that indeed $M_{k}=0$ and $a_{k} \geqq 2 \pi$ ). The existence of two different quadratic differentials compatible with a set of constants associated to a set of curves is in contradiction with the uniqueness of Theorem 5.1. Thus the polyhedron equivalent to the Feynman graph with internal lines cannot be restricted and must therefore have at least one closed critical trajectory of length less than $2 \pi$.

The Four-Punctured Sphere. There are four Feynman graphs associated with this moduli space. Let us now show that no graph overcounts surfaces nor produces surfaces given by another graph. We also show that any surface must be in one of these graphs.

Recall the Strebel presentation of the moduli space of the four-punctured sphere. All surfaces can be presented as a contact interaction which has a tetrahedron as the critical graph. This tetrahedron is parametrized by its edges, with variables $a, b, c$ satisfying $a+b+c=2 \pi$. Edges opposite in the tetrahedron have identical lengths (Fig. 5). The space of possible values for $a, b$, and $c$ is also shown in Fig. 5 (the antiholomorphically related surfaces [SaZw] have been omitted, for simplicity). It is important to note that the shaded regions correspond to the contract interactions. Denote by $l_{n m}$, the length of the shortest closed path surrounding punctures $n$ and $m$. It follows from the figure that:

$$
l_{12}=l_{34}=2(b+c), \quad l_{13}=l_{24}=2(a+c), \quad l_{14}=l_{23}=2(a+b) .
$$

In the pure interaction region all these lengths are greater or equal to $2 \pi$, thus obeying the condition for a restricted tetrahedron. As shown before there cannot be any overlap between surfaces produced in the pure interaction and those produced with intermediate propagators. The necessity of having a tetrahedron 

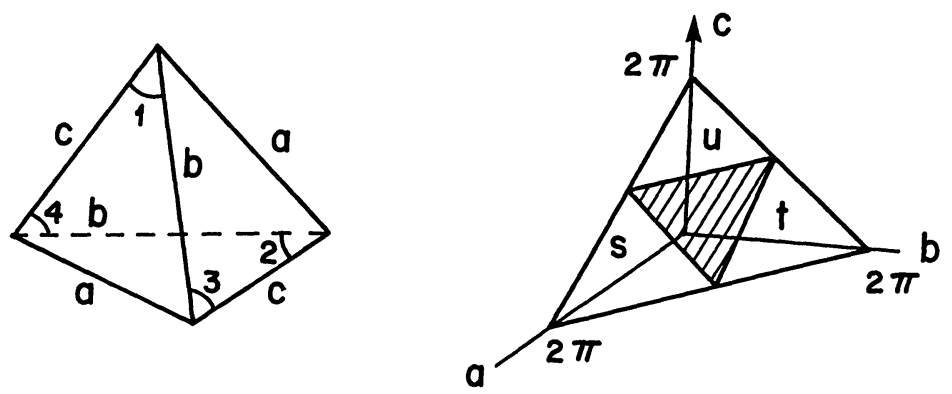

Fig. 5. a The tetrahedron is the polyhedron corresponding to a four-punctured sphere. $\mathbf{b}$ The decomposition of the moduli space of the four-punctured sphere. The shaded region corresponds to restricted tetrahedra. The region denoted by $s$, corresponds to tetrahedra where a closed curve separating punctures $\{1,2\}$ from punctures $\{3,4\}$ has length less than $2 \pi$

type interaction was discussed in [Ka, KaLy], before the polyhedral approach to classical closed string theory was developed.

Consider now the region of $\{a, b, c\}$ space indicated by (s) in the Fig. 5. Here $l_{12}<2 \pi$ and both $l_{13}$ and $l_{14}$ are greater than $2 \pi$. It follows that the corresponding polyhedron does not maximize the functional $\left(\sum M_{j}+M_{12}\right)$, where $M_{12}$ is the modulus of an intermediate annulus homotopic to $l_{12}$. The quadratic differential that maximizes this functional must have an intermediate annulus, thus it must correspond to an $s$-channel Feynman graph.

Can a surface be produced two or more times by the s-channel Feynman graphs? It cannot. The reason is the following. If there is overcounting then we have two different quadratic differentials for the same surface. Because of uniqueness they must correspond to two inequivalent admissible curves separating punctures $\{12\}$ from punctures $\{14\}$. Since both curves give rise to a quadratic differential with an intermediate annulus they must both have lengths smaller than $2 \pi$ when seen on the corresponding polyhedron. This, however, is impossible. Take the one of lowest length. The other curve must be obtained from the first one by a Dehn twist, but a Dehn twist performed around any of the other closed cycles on the polyhedron (both of which are of length greater than $2 \pi$ ) must add a length greater than $(4 \pi)$. As a consequence the second cycle must have a length greater than $2 \pi$. This proves the absence of overcounting within this channel (or within any other channel).

Can there be overlap between the different channels? No, a surface in the $s$-region of $\{a, b, c\}$ space does not have, in the Strebel polyhedron a closed curve separating punctures $\{14\}$ and $\{23\}$, appropriate for a $t$-channel graph, with length less than $2 \pi$. Thus it cannot be represented as a $t$-channel graph.

We have seen that classical closed string theory chooses different quadratic differentials for the various regions of $(a, b, c)$ space. In fact, we can now see in this simple example that the chosen quadratic differential is that for which the sum of all moduli (reduced and ordinary) is maximal. Introducing an intermediate annulus diminishes the sum of reduced moduli (since the punctured disks must reduce) but may increase the overall sum of moduli. For a surface $R$ in the $s$-region of $\{a, b, c\}$ space we have shown that there is a unique admissible curve with length less than $2 \pi$. For any other choice of curve the quadratic differential must be the Strebel one, by uniqueness. Therefore, there are only two candidate quadratic 


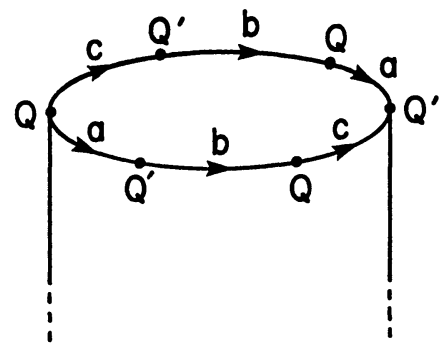

Fig. 6. The polyhedron associated with a once-punctured torus. Here $a+b+c=\pi$, and the edges should be identified along the arrows. The points $Q$ and $Q^{\prime}$ are two first order zeroes of the quadratic differential

differentials for the surface $R$, a Strebel one, called $\phi_{s}$, corresponding to a tetrahedron with a closed cycle smaller than $2 \pi$, and a quadratic differential $\phi_{i}$, with an intermediate annulus. It follows that $\phi_{i}$ maximizes

$$
\mathscr{F}_{i}=\sum M_{j}+M_{k} ; \quad \mathscr{F}_{i}\left(\phi_{i}\right)=c_{i},
$$

and that $\phi_{s}$ maximizes

$$
\mathscr{F}_{s}=\sum M_{j} ; \quad \mathscr{F}_{s}\left(\phi_{s}\right)=c_{s},
$$

where $c_{i}$ and $c_{s}$ are some constants. Because of its cycle of length less than $2 \pi, \phi_{s}$ does not maximize $\mathscr{F}_{i}$. Therefore $\mathscr{F}_{i}\left(\phi_{s}\right)<c_{i}$, but since $\phi_{s}$ has no intermediate annulus $\mathscr{F}_{i}\left(\phi_{s}\right)=\mathscr{F}_{s}\left(\phi_{s}\right)=c_{s}$. We therefore have $c_{s}<c_{i}$, and indeed, by choosing to use $\phi_{i}$ in the $s$-region of $(a, b, c)$ space, closed string theory is using the quadratic differential with the largest possible sum of moduli.

The Once Punctured Torus. This case is also easily dealt with because, as was the case in the four-punctured sphere, there is at most one curve in any admissible set. Consider the polyhedron representation of this surface, as shown in Fig. 6. The sides of lengths $a, b, c$ must be glued as indicated by the arrows (this representation was explained at length in [SoZw 2]). Since the closed horizontal trajectories around the puncture must be of length $2 \pi$, one must have $a+b+c=\pi$. The space $\{a, b, c\} \geqq 0$ with the constraint on the sum gives all once-punctured tori exactly three times [SoZw 2].

The string amplitude corresponding to a once punctured torus is a one-loop tadpole. For this string amplitude, the naive Feynman rules lead to string diagrams that are well known to give serious overcounting of surfaces [ZZ]. The string diagram corresponds to a JS quadratic differential with one punctured disk and one annulus, both with closed horizontal trajectories of length $2 \pi$. The overcounting can be explained now. For any once-punctured torus there are several nonhomotopic closed curves with lengths less than $2 \pi$ in the corresponding polyhedron. For example $a+b, a+c$, and $b+c$, are the lengths of closed curves shorter than $2 \pi$, since $a+b+c=\pi$. Each different choice of a curve shorter than $2 \pi$ can produce a different quadratic differential for the same surface giving rise to the overcounting. It is interesting to note that the number of nonhomotopic curves of length less than $2 \pi$ on the polyhedron is finite for every surface, and becomes larger as the surface approaches degeneration. Thus the naive one loop tadpole does not produce any (nondegenerate) surface an infinite number of times. One may calculate, using the above ideas what is the maximal number of times any surface can appear. 


\section{The Generalized Extremal Problem}

It was illustrated in the previous section that the quadratic differential chosen to represent a particular four punctured sphere was that which would maximize the sum of moduli. It will be seen now that this is the case in general. It will be established that classical closed string theory solves the following problem for the particular case of Riemann spheres with $n \geqq 3$ punctures.

Extremal Problem 7.1. Given a genus $g$ Riemann surface $R$ with $n \geqq 0$ punctures ( $n \geqq 3$ for $g=0$ ) and a fixed set of local coordinates at the punctures, consider the set $\Gamma_{R}=\left\{\gamma_{1}, \gamma_{2}, \ldots\right\}$, whose elements $\gamma_{i}$ are all possible admissible sets of curves for the surface. For each admissible set $\gamma_{i} \in \Gamma_{R}$ one can maximize the corresponding functional:

$$
\mathscr{F}_{\gamma_{i}}=(2 \pi)^{2}\left(\sum M_{j}+\sum M_{k}\right)
$$

where $M_{j}$ are the reduced moduli of the punctured disks (with respect to the fixed coordinates at the punctures), and $M_{k}$ are the moduli of the annuli homotopic to the curves in $\gamma_{i}$. The maximum is attained by the ring domains of a unique quadratic differential $\phi_{\gamma_{i}}$. Denote the maximum value of $\mathscr{F}_{\gamma_{i}}$ by $\mathscr{F}\left(\phi_{\gamma_{i}}\right)$. This is just the (reduced) norm of the quadratic differential $\phi_{\gamma_{i}}$.

In this way we find a collection $\Phi_{R}=\left\{\phi_{\gamma_{1}}, \phi_{\gamma_{2}}, \ldots\right\}$ of quadratic differentials, and an associated collection of (reduced) norms $\mathscr{F}_{R}=\left\{\mathscr{F}\left(\phi_{\gamma_{1}}\right), \mathscr{F}\left(\phi_{\gamma_{2}}\right), \ldots\right\}$.

The extremal problem consists of finding among the quadratic differentials of $\Phi_{R}$ that (those) quadratic differential( $s$ ) of largest norm.

Let us first show that all elements in $\mathscr{F}_{R}$ are bounded by a single number $\bar{M}$, and therefore the maximal possible norm is not infinity. Consider the punctured disks, there are $n$ of them and the reduced modulus of each of them is bounded. Let $\bar{m}$ denote the largest of those bounds. It follows that $\sum_{j} M_{j}$ is bounded by $n \bar{m}$. Now consider the internal annuli. There are at most $(3 g-3+n)$ of them. It is shown in [Ga] that the modulus $M_{k}$ of an annulus homotopic to a curve $\gamma_{k}$ is bounded by $\pi / l(k)$, where $l(k)$ is the length (in the hyperbolic metric) of the geodesic homotopic to $\gamma_{k}$ on the Riemann surface. Let $l$ denote the shortest geodesic on the surface (not homotopic to a puncture). Then the modulus of every annulus is bounded by $\pi / l$, and therefore $\sum M_{k}$ is bounded by $\pi(3 g-3+n) / l$. It follows that $\bar{M}=n \bar{m}$ $+\pi(3 g-3+n) / l$ is an upper bound for all the elements in $\mathscr{F}_{R}$.

It should also be established that the maximal norm is attained by a (various) quadratic differential(s) in $\Phi_{R}$ and is not just the limit of a sequence. ${ }^{8}$ We will consider the case when there is such infinite sequence, and show that the limit of the norm is attained by some quadratic differential(s) in $\Phi$. Consider an infinite sequence of quadratic differentials and associated norms. By assumption the quadratic differentials in this sequence are not equal (and actually may vary greatly from one element to the next) but their norms are going to the limit. Since each quadratic differential can be associated with a Feynman graph, at least one Feynman graph must be appearing an infinite number of times in this sequence. We consider now the infinite subsequence of quadratic differentials corresponding to this particular Feynman graph. In this subsequence the quadratic differentials

${ }^{8}$ I thank G. Zemba for a discussion on this point 
might still vary considerably from one element to the next, but their norms are going to the limit. Each element has the same number of internal annuli corresponding to some curves $\left\{\gamma_{k}\right\}$, and the quadratic differential arises by maximization of $\sum M_{j}+\sum M_{k}$. Since given a curve system there is a unique quadratic differential, the infinite sequence of quadratic differentials corresponds to an infinite sequence of admissible sets of curves all related to $\left\{\gamma_{k}\right\}$ by Dehn twists (which do not change the Feynman graph, see Sect. 2.3). It follows that in this sequence of admissible curves the lengths of all the curves cannot be bounded; this is so because there is only a finite number of sets of curves with all their lengths bounded. So, at least one of the curves in $\left\{\gamma_{k}\right\}$ must be growing infinitely long. Assume all of the curves are growing infinitely long, then it follows that $\sum M_{k}$ must be going to zero, and the norm of the quadratic differentials is converging to $\mathscr{F}=\sum M_{j}$, which is attained by the Strebel quadratic differential corresponding to the polyhedron associated to the surface. If only a subset of the curves in $\left\{\gamma_{k}\right\}$ is becoming infinitely long, consider the curves $\left\{\gamma_{k}^{(b)}\right\}$ that remain bounded. They make an admissible set, and there are only a finite number of such sets, because of the boundedness. The infinite sequence of admissible curves can now be broken into a finite number of subsequences, each one corresponding to a $\left\{\gamma_{k}^{(b)}\right\}$. Some of these sequences may be finite (and irrelevant since they may not attain the limit) but at least one must be infinite. The norms of the quadratic differentials in this subsequence converge to the maximal value. But the annuli corresponding to the curves that are becoming infinitely long must collapse, giving, in the limit a norm which is just the maximal value of $\mathscr{F}=\sum M_{j}+\sum M_{\gamma_{k}^{(b)}}$, which is attained by the quadratic differential corresponding to the admissible set $\gamma_{k}^{(b)}$. This shows that the maximal norm is attained by some element of $\Phi$.

It seems plausible, given the examples of the previous section that, in fact, the set $\Phi_{R}$ actually contains a finite number of different quadratic differentials for any nondegenerate Riemann surface $R$. Let us now establish some properties of the quadratic differential of maximal norm in $\Phi_{R}$.

Theorem 7.2. Given a genus $g$ Riemann surface $R$ with $n \geqq 0$ punctures ( $n \geqq 3$ for $g=0)$ a quadratic differential in $\Phi_{R}$ of maximal norm satisfies the following properties:

(i) The singularities are $n$ second order poles at the punctures,

(ii) The characteristic ring domains are $n$ punctured disks and $k$ annuli, where $0 \leqq k$ $\leqq 3 g+n-3(k=1$ for $g=n=0)$,

(iii) The lengths of the closed horizontal trajectories on the punctured disks and annuli are all equal to $2 \pi$,

(iv) Every nontrivial closed critical trajectory has length (measured using the quadratic differential) greater or equal to $2 \pi$.

Proof. Note that the above conditions are almost the same as those of Theorem 3.2 for the classical theory, namely Riemann spheres. Here in the present theorem, we do not discuss uniqueness. Conditions (i), (ii), and (iii) are clearly satisfied by all the quadratic differentials in the set $\Phi_{R}$ due to the way the functionals $\mathscr{F}$ in Eq. (7.1) are defined. Condition (i) is satisfied because the functionals include $n$ reduced moduli, condition (ii) is satisfied because the maximal number of admissible curves is $3 g+n-3$, and condition (iii) is satisfied because all functionals have $A_{i}=(2 \pi)^{2}$ for all moduli.

Condition (iv) is the nontrivial one and requires that the quadratic differential be one of maximal norm. It implies that every critical graph is a restricted 
polyhedron (classical or quantum). Imagine that condition (iv) is violated, namely, there is some closed $\gamma_{k^{\prime}}$ path on a polyhedron whose length is smaller than $2 \pi$. Denote by $\left\{\gamma_{k}\right\}$ the set of curves homotopic to the propagators. It is clear that the Feynman graph maximizes the functional $\mathscr{F} \sim\left(\sum M_{j}+\sum M_{k}\right)$. However, the extra curve $\gamma_{k^{\prime}}$ is compatible with the curves $\left\{\gamma_{k}\right\}$. It follows that the given Feynman graph does not maximize the functional $\mathscr{F}+M_{k^{\prime}}$ corresponding to the admissible set of curves including $\left\{\gamma_{k}\right\}$ and $\gamma_{k^{\prime}}$. This is in contradiction with the assumption that the quadratic differential maximizes $\mathscr{F}$ for all possible choices of admissible curves. It follows that no closed path on a polyhedron can have length smaller than $2 \pi$, and therefore all polyhedra must be restricted and condition (iv) is obeyed. This concludes the proof of this theorem.

The physical implication of the above result is that all surfaces (including unpunctured higher genus ones) can be constructed by the naive Feynman rules of a closed string field theory that includes all restricted polyhedra (classical and quantum). This is so because, by definition, the naive Feynman rules construct all quadratic differentials satisfying properties (i) to (iv), and, due to the above theorem, to any surface we can associate at least one quadratic differential (that of maximal norm) satisfying properties (i) to (iv).

Let us now turn to the case when the Riemann surface is a sphere with three or more punctures, and consider the extremal problem 7.1. We will show that in this case there is a unique quadratic differential in $\Phi_{R}$ which has maximal norm, and that this quadratic differential defines a string diagram which is nothing else than the string diagram constructed by classical closed string field theory. We will establish this by giving now a proof of Theorem 3.2.

Proof of Theorem 3.2. (a) The existence is now a simple consequence of Theorem 7.2. Take a quadratic differential of maximal norm in $\Phi_{R}$, where $R$ is the punctured Riemann sphere $(n \geqq 3)$. The properties listed in Theorem 7.2, become, for this case, those listed in Theorem 3.2.

(b) We now show that any quadratic differential $\phi$ satisfying the conditions of Theorem 3.2, is unique. This proves the uniqueness part of Theorem 3.2, and also, due to (a), that the above extremal problem, that of choosing the quadratic differential of largest norm, furnishes a unique quadratic differential for the case of punctured Riemann spheres. The uniqueness does not rule out the possibility that two different admissible sets do give rise to the same quadratic differential $\phi$ with maximal norm.

The idea will be to show that all candidate quadratic differentials solve the same minimal area problem. The uniqueness of the extremal area metric will allow us to show that the quadratic differentials are actually the same.

Consider a quadratic differential satisfying the conditions of Theorem 3.2. We know that all closed curves homotopic to those in the associated admissible set have lengths greater or equal to $2 \pi$. We have seen that all closed critical trajectories also have lengths greater or equal to $2 \pi$. It now follows that any homotopically nontrivial curve on the surface will have length greater or equal to $2 \pi$. The reason is that the remaining nontrivial curves must go through annuli joining disconnected critical graphs, as shown in Fig. 7. Imagine collapsing all the involved annuli, namely letting $T \rightarrow 0$ in all those propagators. In doing this the curve becomes shorter and turns into a closed curve on the single critical graph obtained by the collapse of the various disconnected critical graphs involved. Since the original disconnected critical graphs were restricted polyhedra, the collapsed graph is also 


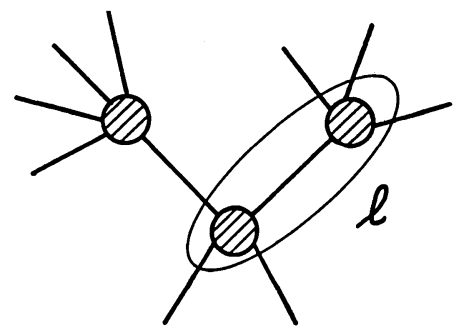

Fig. 7. In a tree level Feynman graph the closed curve indicated above must satisfy $l \geqq 2 \pi$

a restricted polyhedron, by the continuity argument of [KKS] (reviewed in Sect. 3.1). Since any closed curve on a restricted polyhedron exceeds length $2 \pi$ the original curve must also exceed length $2 \pi$, as stated.

The fact that any closed curve has length greater than $2 \pi$ has enormous implications. It follows that the above quadratic differential is the solution to the following generalized minimal area problem: given a punctured Riemann sphere find the metric that minimizes the (reduced) area given that all nontrivial homotopy closed curves have lengths greater or equal to $2 \pi$. A priori, there is no reason why the solution to this problem should involve a quadratic differential. We know that specifying minimal lengths for curves homotopic to some admissible set leads to an extremal metric given by the quadratic differential (Theorem 5.2). But the set of all nontrivial closed curves on a surface is not an admissible set! Even though one is not guaranteed a priori that the solution is a quadratic differential, one may try it. The idea is the following. Pick an admissible set, all curves homotopic to them must have lengths greater than $2 \pi$. Under this condition, a quadratic differential gives the lowest area metric. One is not guaranteed that other closed curves are longer than $2 \pi$, but if the quadratic differential satisfies the conditions of Theorem 3.2, it will happen, as was shown above. Then the metric given by the quadratic differential actually minimizes the area under the condition that all curves be longer than $2 \pi$. Suppose this is not true, then there would exist another metric giving smaller area but still being compatible with the particular admissible set, in direct contradiction with the fact that the quadratic differential gives the lowest possible area for a metric compatible with the admissible set.

It has been shown that a quadratic differential which satisfies the conditions of Theorem 3.2 actually solves the problem of finding the minimal area metric for which any closed curve in the surface has length greater than $2 \pi$. As we have shown in Sect. 2.1, this extremal metric must be unique. This implies that if two quadratic differentials $\phi$ and $\psi$ are solutions of the extremal problem, we must have $|\phi|=|\psi|$ almost everywhere. Since quadratic differentials are analytic objects, one must have $|\phi|=|\psi|$ everywhere. ${ }^{9}$ Moreover, since they have equal absolute values they must be (locally) of the form $\phi(z)=e^{i \theta(z)} \psi(z)$. Then $\theta(z)$ must be purely real, and can only be a constant. Thus the two candidate quadratic differentials could only differ by a constant phase. This phase, however, must be zero because we know both quadratic differentials have second order poles with identical residues (real residues, in fact, since otherwise there would not exist closed horizontal trajectories near the punctures). Thus $\phi=\psi$ and this shows the

\footnotetext{
${ }^{9}$ I thank K. Strebel for clarifying to me the above delicate issues
} 
uniqueness of the solution. This finishes the proof of Theorem 3.2. In proving Theorem 3.2 we have also established that:

Theorem 7.3. Consider an n-punctured Riemann sphere. The metric of lowest (reduced) area under the condition that any nontrivial homotopy closed curve in the surface have length greater or equal to $2 \pi$ arises from a unique $J S$-quadratic differential satisfying the conditions of Theorem 3.2.

\section{On Higher Genus String Diagrams}

In this section we try to learn as much as possible about string diagrams for higher genus using all the tools developed so far. The problem is to find a canonical definition for those string diagrams. As we will see, the basic idea is to take the string diagram to be defined by the surface of minimal area under the condition that all nontrivial closed curves be longer than $2 \pi$. We postpone here, to some degree, a discussion of how these diagrams could arise from a field theory. Actually, the discussion below is quite reminiscent of the approach to loops discussed by Sonoda and the author in [SoZw 2], but no effort will be made here to establish a connection. A different approach to loops, based on subtracting counterterms has been explored by Saadi [Sa].

The naive Feynman rules of field theory would indicate that we could build all higher genus punctured Riemann surfaces by gluing restricted classical polyhedra with tubes (propagators) of circumference $2 \pi$. It could be expected that every surface would be produced once and only once. This does not happen. It is wellknown, from the study of the one-punctured torus $[\mathrm{ZZ}, \mathrm{GM}]$ that the naive Feynman rules overcount. We have seen in Sect. 6 that the naive Feynman rules based on the classical action eventually undercount because of the necessity of adding the restricted quantum polyhedra. In fact, the naive Feynman rules, using all restricted polyhedra, classical and quantum, will not miss surfaces as shown after the proof of Theorem 7.2. For any surface there is at least one (and possibly several) naive Feynman graphs. The problem, of course is that while they do not miss surfaces, the naive Feynman rules using all polyhedra do overcount.

For fixed genus $g \geqq 1$, there exist restricted quantum polyhedra when the number of punctures is large enough. It is simple to see that there are no onepunctured restricted quantum polyhedra. This is so because one has a single ring domain and given that there should be some identifications along its edge, which is of perimeter $2 \pi$, closed cycles representing handles will have circumferences smaller than $2 \pi$. Similarly one can see that the $(n=2,3 ; g=1)$ restricted quantum polyhedra vanish too. Finally it is possible to convince oneself that for $g=1$, and sufficiently high $n$ (say $\sim 25$ ) there exist restricted quantum polyhedra.

It is convenient to define restricted Feynman graphs.

Definition. A restricted Feynman graph is a Feynman graph built with restricted polyhedra (classical and quantum) in which any closed curve has length greater or equal to $2 \pi$.

For tree amplitudes every Feynman graph is automatically a restricted one, but this is not the case for loop amplitudes. Since restricted Feynman graphs are built from propagators and restricted polyhedra, closed curves homotopic to ring domains and closed critical trajectories automatically satisfy the length condition; therefore only a small set of curves needs to be checked. These are curves that 


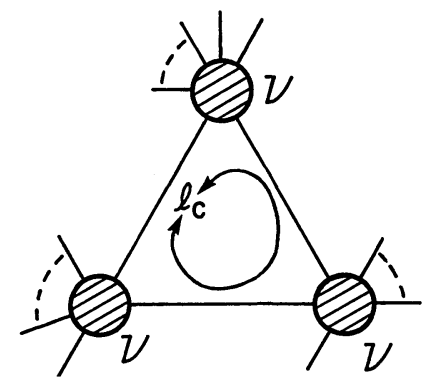

Fig. 8. In a higher genus string diagram a restricted Feynman graph must have all loop curves, such as $l_{c}$ longer or equal to $2 \pi$

correspond to the closed loops in the Feynman graph representation of the string diagram. An example is the curve $l_{c}$ shown in Fig. 8. Let us call these curves loop curves. Thus a restricted Feynman graph is a Feynman graph with all the loop curves longer or equal to $2 \pi$. Restricted Feynman graphs are clearly a well defined notion for vacuum graphs.

Properties of Restricted Feynman Graphs. Let us now show that restricted Feynman graphs have the following significant properties:

(a) Restricted Feynman graphs construct the surfaces of minimal area under the condition that any nontrivial closed curve be longer or equal to $2 \pi$.

(b) Restricted Feynman graphs do not overcount punctured higher genus surfaces.

(c) Restricted vacuum Feynman graphs (with critical closed trajectories and loop curves satisfying the strict inequalities for length) do not overcount higher genus surfaces without punctures.

(d) Restricted Feynman graphs define a system of factorizable quadratic differentials.

Let us see why part (a) is true. A restricted Feynman graph defines a JS quadratic differential giving the minimal area metric under the condition that some curves be longer than $2 \pi$. But since the Feynman graph is restricted, all curves are greater than $2 \pi$ and therefore the metric arising from the quadratic differential solves the generalized minimal area problem. In particular, restricted quantum polyhedra correspond to minimal area metrics. The correctness of $(b)$ is an immediate consequence of (a) and the uniqueness of the minimal area metric. Given two different quadratic differentials one must have $|\phi|=|\psi|$. As argued in the previous section, the two quadratic differentials can only differ by a phase. For the case of punctured surfaces the phase is zero and the quadratic differentials must be identical, showing the absence of overcounting of punctured surfaces.

Let us now consider (c). For surfaces without punctures we need a more detailed argument. Assume there are two different quadratic differentials $\phi$ and $\psi$ for the same surface. The non-degenerate ring domains of the two quadratic differentials must arise from different sets of admissible curves denoted as $\gamma_{\phi}$ and $\gamma_{\psi}$. We will assume that the Feynman graphs are definitely restricted, that is, all closed critical trajectories making the polyhedra and all loop curves are greater than $2 \pi$. Now, since the admissible sets of curves differ, at least some curve $\hat{\gamma}$ in one of the systems must be homotopic to either a critical trajectory or to a loop curve in the 

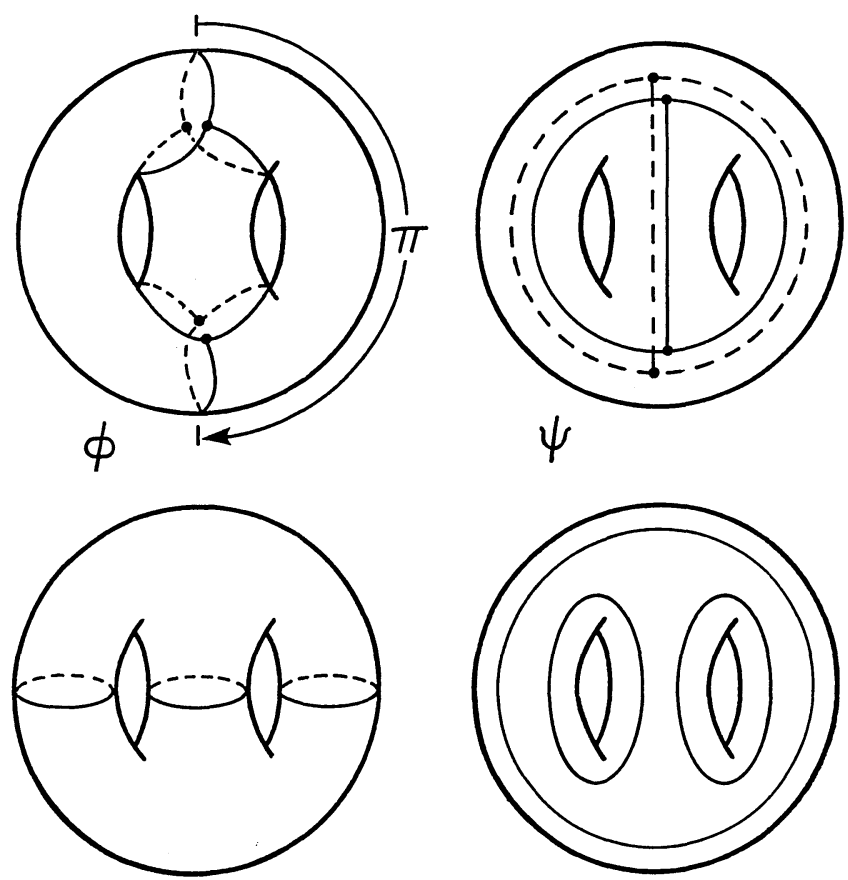

Fig. 9. Upper left: a JS quadratic differential $\phi$ on a genus two surface without punctures. The heights of the three annuli are $\pi$ and the circumferences $2 \pi$. Upper right: The same surface admits another quadratic differential $\psi=-\phi$ with equal heights and circumferences. The corresponding admissible set of curves are shown below

string diagram corresponding to the other system (this is true even if one set of curves is a subset of the other). Since the metrics are the same, curves homotopic to $\hat{\gamma}$ have to be longer than $2 \pi$, contradicting the fact that there should exist an annulus homotopic to $\hat{\gamma}$ with closed horizontal trajectories of circumference $2 \pi$. This proves that all the curves in $\gamma_{\psi}$ should be homotopic to those in $\gamma_{\phi}$, and as a consequence the two quadratic differentials must be identical. The condition that the inequalities must be satisfied is necessary in order to show that the quadratic differentials are identical, otherwise they may indeed differ by a phase. An example of this situation is presented for a genus two surface in Fig. 9. This surface is built using three cylinders each of circumference $2 \pi$ and height $\pi$. The closed critical trajectories of two quadratic differentials $\phi$ and $\psi$ are shown in the figure. The two quadratic differentials are related by $\phi=-\psi$, and both have closed horizontal trajectories of length $2 \pi$. The corresponding sets of admissible curves are shown below, and in fact they correspond to the same Feynman graph configuration. The surfaces produced when closed critical trajectories or loop curves are equal to $2 \pi$ belong to (lower dimensional) boundaries of the regions of moduli space generated by restricted Feynman graphs. Thus any overcounting here has no physical relevance, in fact the above example would not correspond to any overcounting at all because the Feynman graph, and the parameters defining it are the same for both $\phi$ and $\psi$, and therefore the Feynman rules would construct it once.

Finally, consider property (d). Factorization is of fundamental physical interest. It was known that the tree level string diagrams of classical closed string field theory defined a system of factorizable quadratic differentials over the moduli 
spaces of the punctured Riemann surfaces [SoZw 2]. The quadratic differentials of all the restricted Feynman graphs form a system of factorizable quadratic differentials over a much larger space. In addition to including the complete moduli spaces $\mathscr{M}_{0, n}$, they exist over a subspace of every moduli space of the higher genus surfaces with and without punctures. Factorization is the result of sewing, or in other words the use of propagators that include infinitely long tubes in the limit. Degenerate surfaces should only be obtained when tubes become infinitely long. This clearly holds for restricted Feynman graphs, given that all closed curves must exceed $2 \pi$, one cannot ever find degenerate surfaces due to the existence of short geodesics in this metric. All degenerations happen because of long tubes. Given a set of string diagrams it is simple to check for factorization. One simple picks a propagator (annulus) in the Feynman graph, cuts it (say along a closed horizontal trajectory), and each of the tubes is then separately extended to infinity. This procedure of degeneration gives either a single surface with one less handle or two separate surfaces. In both cases this operation induces quadratic differentials in the new surfaces. One has a system of factorizable quadratic differentials if the induced quadratic differentials obtained by degeneration are precisely the quadratic differentials that have been assigned to the corresponding surfaces. This does happen for the restricted Feynman graphs, the result of this operation of degeneration gives either a single surface or two surfaces that correspond to restricted Feynman graphs. This is so because as the result of opening up a handle one cannot possibly introduce a curve shorter than $2 \pi$ in the resulting surfaces. Rigorously speaking, in order that the restricted Feynman graphs define a factorizable set of quadratic differentials one must add a quadratic differential for the twice-punctured sphere. This is obtained by factorization of the one loop vacuum graph. Thus we assign to the two punctured sphere a quadratic differential with two second order poles of equal negative residues making the closed horizontal trajectories of length $2 \pi$. This is just an infinitely long tube of circumference $2 \pi$. One may wish to think of a circle of circumference $2 \pi$ as a twofaced restricted polyhedron.

It seems clear, however, that restricted Feynman graphs are not the complete story for higher genus surfaces. It has not been established that the restricted Feynman graphs produce all surfaces. It is extremely curious that restricted Feynman graphs produce exactly the correct one loop vacuum graph (one-loop cosmological term). Given a cylinder of circumference $2 \pi$ length $l$ and twist angle $-\pi \leqq \theta \leqq \pi$, the condition that closed paths be of length greater or equal to $2 \pi$ on the torus obtained by gluing the two boundaries of the cylinder requires $l^{2}+\theta^{2}$ $\geqq(2 \pi)^{2}$. Given that $\tau=(\theta+i l) / 2 \pi$, and that the angle condition gives $-1 / 2 \leqq \operatorname{Re}(\tau)$ $\leqq 1 / 2$, the above length constraint which implies $|\tau| \geqq 1$, is precisely the missing condition that singles out the modular region. Despite this encouraging result for the vacuum graph, it seems to follow from the explicit results of [ZZ], that restricted Feynman graphs do not produce all the $g=n=1$ Riemann surfaces. In particular we seem to be missing the once punctured $\tau=i$ torus! Presumably all the missing higher genus surfaces should be represented by string diagrams defined by metrics solving the generalized minimal area problem. It is not clear that the minimal area metrics for these surfaces will arise from JS quadratic differentials although it would seem somewhat odd in view of the results in this paper if they did not. This important question should be answered. Some unsuccessful attempts by the author have led him to believe that perhaps one may have first order poles in the quadratic differentials. A first order pole in a quadratic differential does not correspond to a semiinfinite tube (as punctures do). It corresponds to a curvature 
singularity in which there is a defect angle of $\pi$. These poles would have appeared had we tried to give canonical metrics to the once-punctured and unpunctured Riemann spheres. For the one punctured sphere one must have one second order pole and two first order poles. This would correspond to a semiinfinite cylinder of circumference $2 \pi$ with the circle which is not at infinity closed off, creating in this way two singular points that correspond to the first order poles. In fact, a path integral on this surface is really what one means in closed string field theory for the operation of $\int \Psi$ which associates to a string state a number (all this in complete analogy with the case for open strings as defined in [Wi]). Finally for the sphere with no punctures one may use a cylinder of circumference and height $2 \pi$ closed off at both boundaries. This would correspond to a quadratic differential with four first order zeroes, located at positions whose cross-ratio is $(-1)$.

\section{Comments}

In this paper the possibility that a generalized minimal area problem is the geometrical basis of covariant closed string field theory has been explored. The most important issue to resolve at this stage appears to be that of finding the minimal area metric for the surfaces that are not generated by the restricted Feynman graphs. This would enable us to address conclusively the quantum part of the string action. It would furnish us with a set of completely factorizable string diagrams which could be helpful in establishing the finiteness of perturbative string theory. On the mathematical side, it may allow to extend the cell decomposition found for the moduli spaces of Riemann spheres, into a cell decomposition for all moduli spaces of the higher genus punctured and unpunctured surfaces.

There are a few additional questions that have been left open. One concerns establishing if for any surface $R$, the set $\Phi_{R}$, defined in Sect. 7 contains a finite number of different quadratic differentials. If this is the case it would imply that the naive Feynman rules never produce a surface an infinite number of times. We have also not tried to give a proof of factorization from the minimal area problem directly, our arguments were heuristic (see $[\mathrm{Zw}]$ ). For the case of restricted Feynman graphs it was simple to prove factorization because the metrics arise from quadratic differentials. Finally, we have not studied which is, for any $g$, the lowest value of $n$ for which there is a restricted quantum polyhedron.

As this work has confirmed, the nontriviality of string field theory lies in that it captures the information about all Riemann surfaces in an action principle. In this action principle, the nature of the interactions is dictated by the Riemann surfaces, while the kinetic operator $Q$ and the arguments of the string field are dictated by a conformal field theory. A string field theory should be capable of a nonperturbative definition of quantum gravity in two dimensions [DS, GrMi, BK] where Riemann surfaces play a prominent role. One way of making progress toward a nonperturbative formulation would be formulating the theory in a background independent way (a recent discussion of this problem, for the classical closed string theory, can be found in [Se].)

The elegance of the minimal area problem underlying the perturbative string field theory suggests that there should be a deep geometrical significance to the series of interactions defining the theory. Since actions are minimal principles, it is tempting to speculate that the minimal principle for the Lagrangian of closed string field theory could be related to the minimal principle defining its string diagrams. 
Acknowledgements. I am grateful to Frederick Gardiner, Kurt Strebel and Michael Wolf for instructive conversations.

\section{References}

[Ah] Ahlfors, L.V.: Conformal Invariants, topics in geometric function theory. New York: McGraw-Hill 1973

[BK] Brezin, E., Kazakov, V.A.: Exactly solvable field theories of closed strings. Phys. Lett. 236 B, 144 (1990)

[Ca] Carlip, S.: Quadratic differentials and closed string vertices. Phys. Lett. B 214, 187 (1988)

[DS] Douglas, M.R., Shenker, S.H.: Strings in less than one dimension. Nucl. Phys. B 335, 635 (1990)

[Ka] Kaku, M.: Geometric derivation of string field theory from first principles: closed strings and modular invariance. Phys. Rev. D 38, 3052 (1988)

[KaLy] Kaku, M., Lykken, J.: Modular invariant closed string field theory. Phys. Rev. D 38, 3067 (1988)

[KKS] Kugo, T., Kunitomo, H., Suehiro, K.: Non-polynomial closed string field theory. Phys. Lett. 226 B, 48 (1989)

[KS] Kugo, T., Suehiro, K.: Nonpolynomial closed string field theory: action and gauge invariance. Nucl. Phys. B 337, 434 (1990)

[Ga] Gardiner, F.: Teichmüller theory and quadratic differentials. New York: John Wiley 1987

[GiMa] Giddings, S., Martinec, E.: Conformal geometry and string field theory. Nucl. Phys. B 278, 91 (1986)

[GrMi] Gross, D.J., Migdal, A.: Nonperturbative two dimensional quantum gravity. Phys. Rev. Lett. 64, 127 (1990)

[Ha] Harer, J.L.: The cohomology of the moduli space of curves. C.I.M.E. lectures

[Je] Jenkins, J.A.: On the existence of certain general extremal metrics. Ann. Math. 66, 440 (1957)

On quadratic differentials whose trajectory structure consists of ring domains. Lectures Notes in Pure and Appl. Math., Vol. 36, pp. 65-70. Sanford S. Miller (ed.)

[Sa] Saadi, M.: On closed string counterterms. Mod. Phys. Lett. A 5, 551 (1990)

[SaZw] Saadi, M., Zwiebach, B.: Closed string field theory from polyhedra. Ann. Phys. 192, $213(1989)$

[SoZw 1] Sonoda, H., Zwiebach, B.: Covariant closed string theory cannot be cubic. Nucl. Phys. B 336, 185 (1990)

[SoZw 2] Sonoda, H., Zwiebach, B.: Closed string field theory loops with symmetric factorizable quadratic differentials. Nucl. Phys. B 331, 592 (1990)

[St 1] Strebel, K.: Quadratic differentials. Berlin, Heidelberg, New York: Springer 1984

[St 2] Strebel, K.: On quadratic differentials with closed trajectories and second order poles. J. d'Analyse Math. XIX, Festband für L.V. Ahlfors 373 (1967)

On quadratic differentials with closed trajectories on open Riemann surfaces. Commentationes in honorem Rolf Nevanlinna LXXX annos nato 1975. Ann. Acad. Sci. Fenn. A.I.2 533 (1976)

[Te] Teichmüller, O.: Untersuchungen über konforme and quasikonforme Abbildungen. Deutsche Math. 3, 621 (1938)

[Wi] Witten, E.: Noncommutative geometry and string field theory. Nucl. Phys. B 268, 253 (1986)

[Zw] Zwiebach, B.: Consistency of closed string polyhedra from minimal area. Phys. Lett. B 241, 343 (1990)

[ZZ] Zemba, G., Zwiebach, B.: Tadpole graph in covariant closed string field theory. J. Math. Phys. 30, 2388 (1989) 